
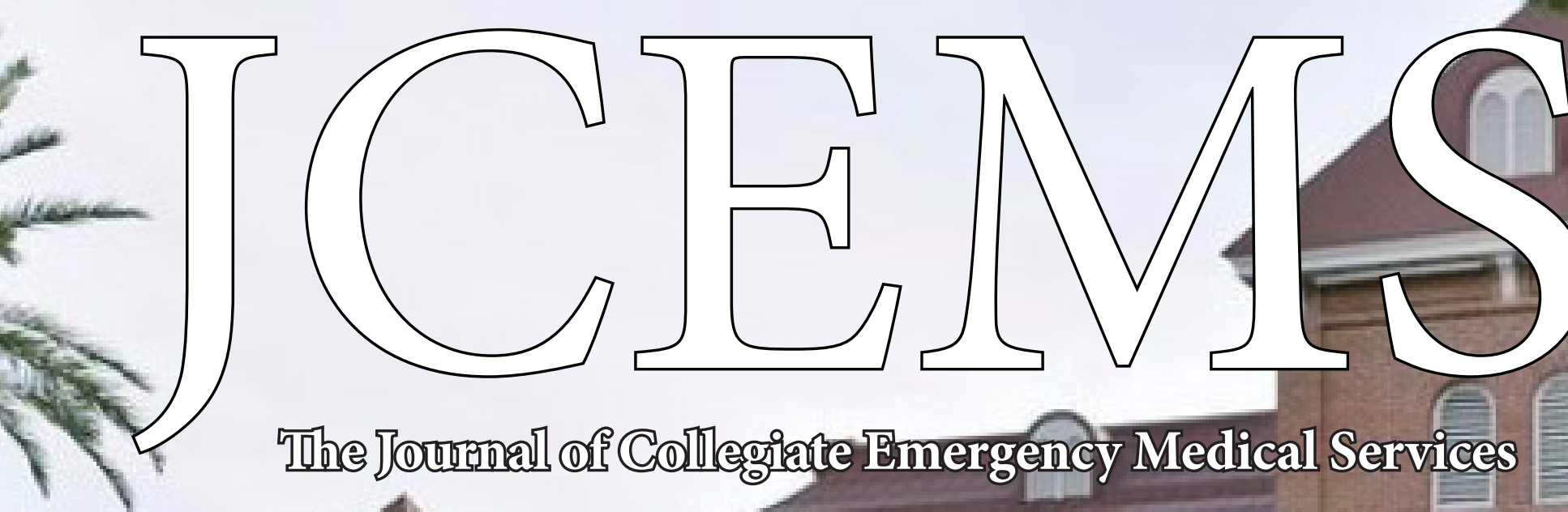

The jowrinal of Collegionte Imergency Meclical Services

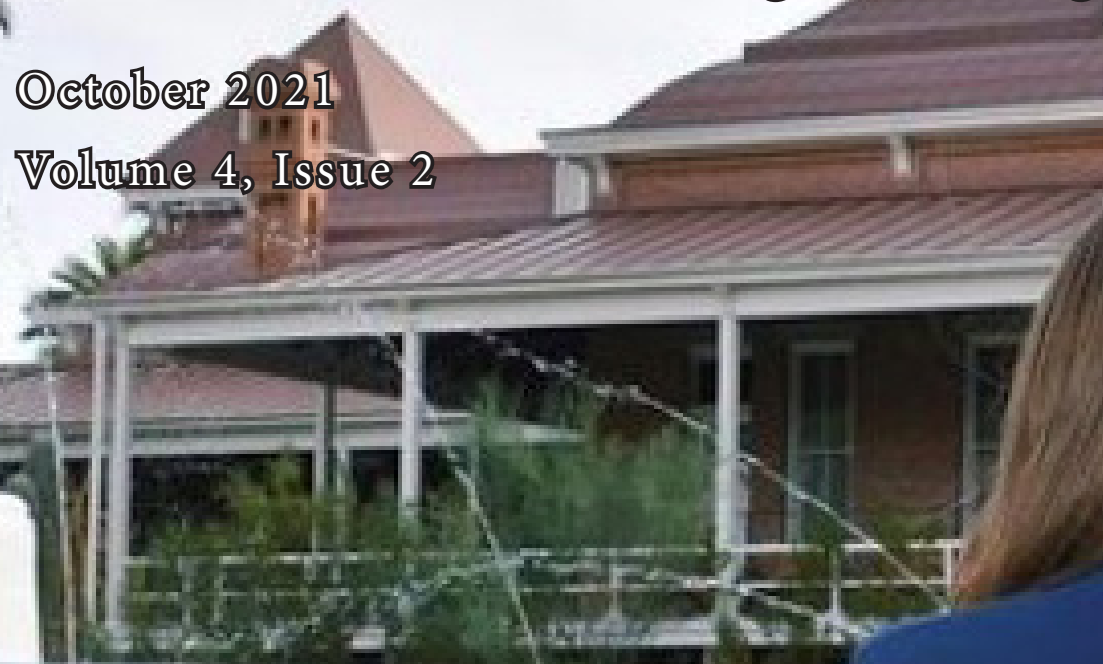

www.CollegeEMS.eom

Voltwe 4, Issue 2

t)
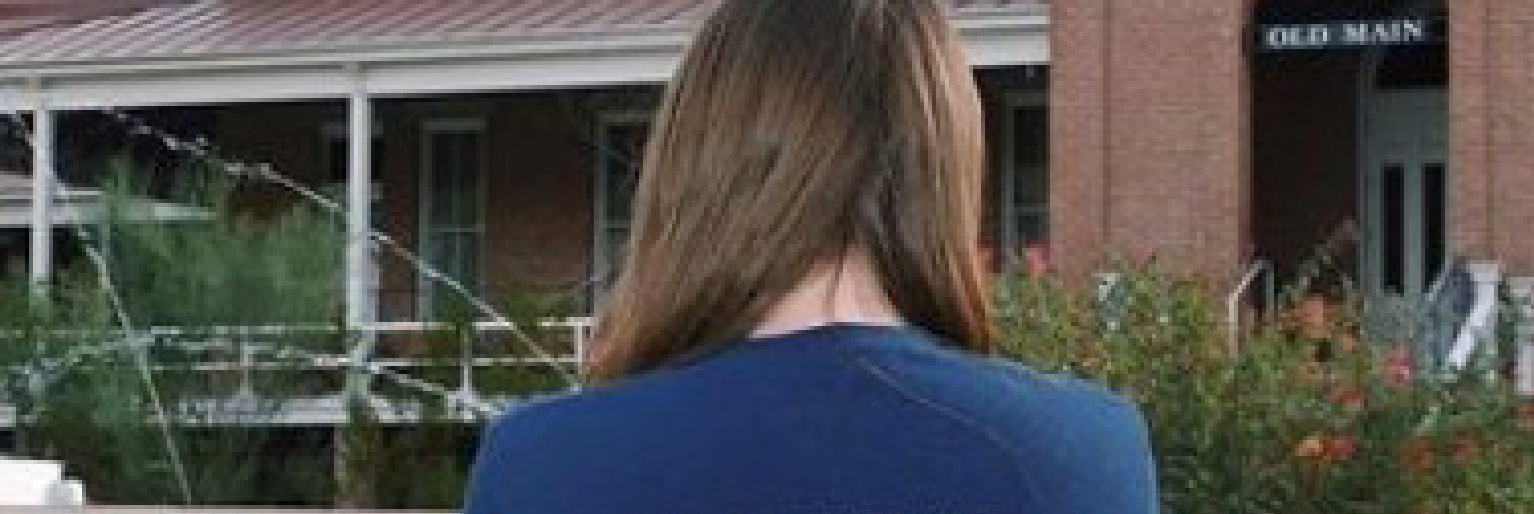

r.

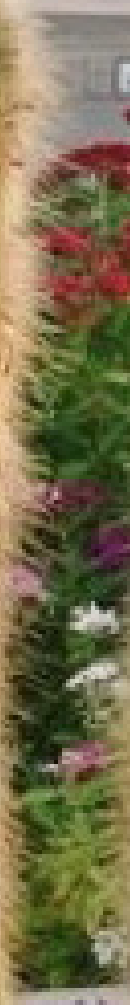

Carompus Divis Pretocol for Mental Health Complaints

2. Actvices Establishing a Collegiate Emergency Medical Service Determinanits of Völunteer Collegiate EMS Budget Size Research orn IMIS Oral Handoff Reports bes

The Official Peer-Reviewed Journal of the National Collegiate Emergency Medical Services Foundation

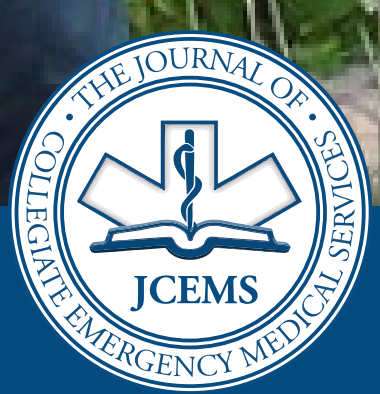




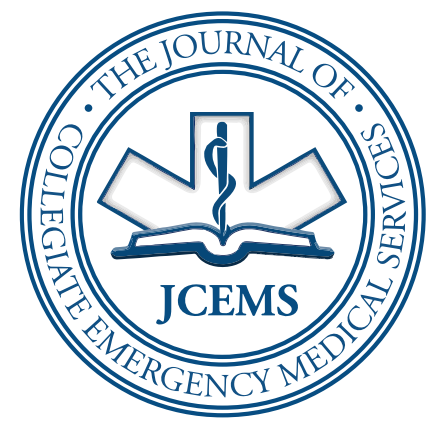

The Journal of COLLEGIATE EMERGENCY MEDICAL SERVICES

The Official Journal of the National Collegiate Emergency Medical Services Foundation

\section{TABLE OF CONTENTS}
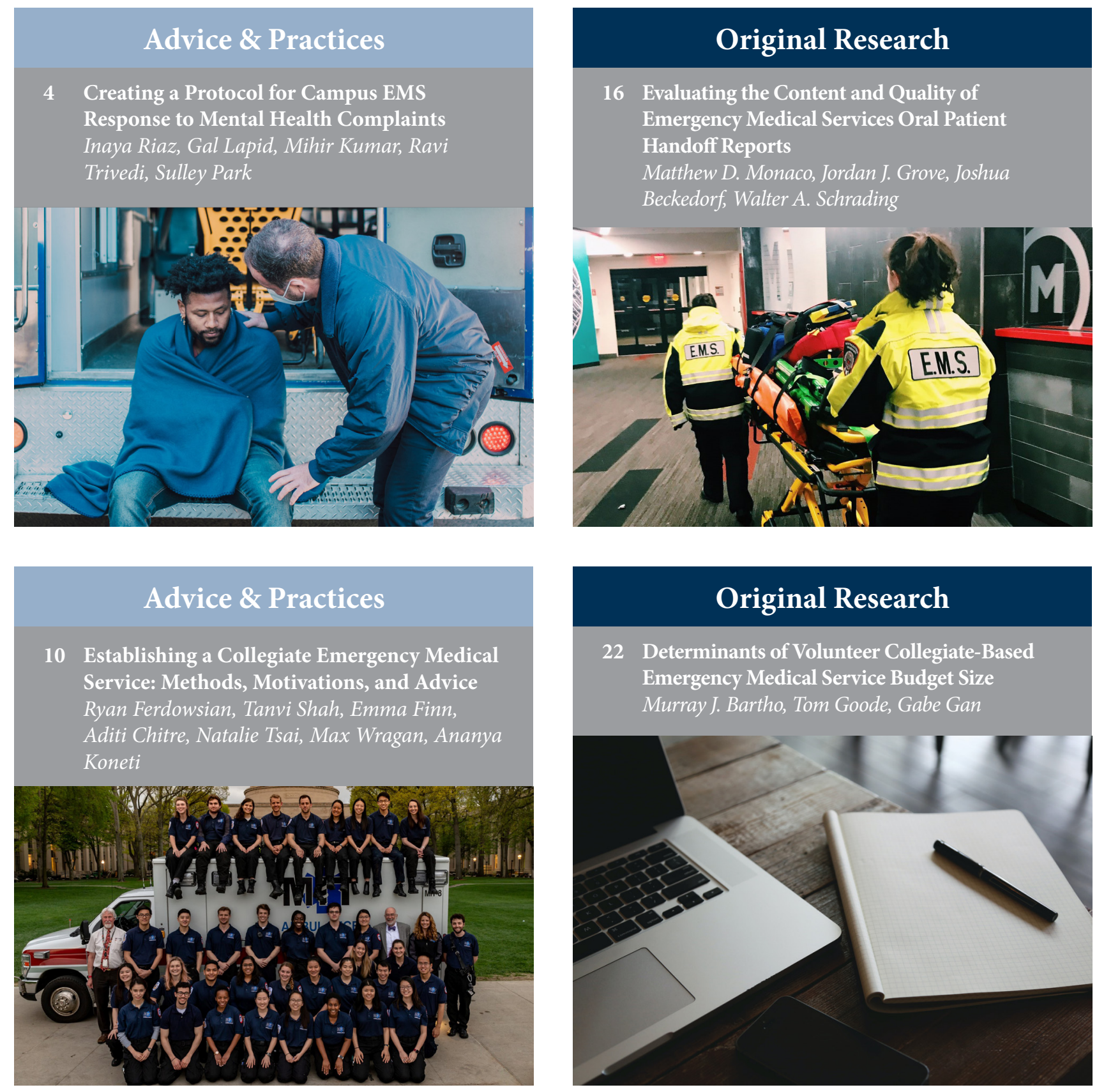

Photo Credits: University of Arizona Emergency Medical Services, Northeastern University Emergency Medical Services, Massachusetts Institute of Technology Emergency Medical Services 


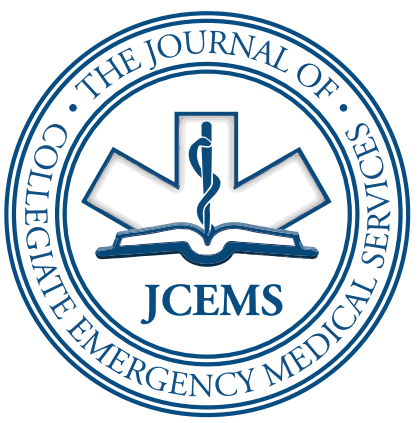

\section{The Journal of COLLEGIATE EMERGENCY MEDICAL SERVICES}

The Official Journal of the National Collegiate Emergency Medical Services Foundation

PUBLICATION INFORMATION

Editor

Christopher Gaeta, EMT-B • Editor-in-Chief

Editorial Board

Jose Victor L. Nable, MD, MS, NRP

Georgetown University School of Medicine, Washington, DC

Nicholas M.G. Friedman, BA, EMT

Stanford University School of Medicine, Stanford, CA

Matthew J. Levy, DO, MS, FACEP

The Johns Hopkins University School of Medicine, Baltimore, MD

Benjamin J. Lawner, DO, MS, EMT-P, FACEP

Temple University School of Medicine, Philadelphia, PA

David Goroff, MS, NRP

New Castle County EMS, New Castle, DE

Albert Jagoda, MD

Skidmore College, Saratoga Springs, NY

Brent Campbell, BA, AEMT-CC

Ambulance Service of Fulton County, Gloversville, NY

Patricia Bosen, MSN, FNP-C

Skidmore College, Saratoga Springs, NY

Joseph M. Grover, MD

University of North Caroline School of Medicine, Chapel Hill, NC

Lauren N. Gorstein, BA, EMT-B

Skidmore College, Saratoga Springs, NY

Michael W. Dailey, MD, FACEP, FAEMS

Albany Medical College, Albany, NY

Jeffrey S. Lubin, MD, MPH, FACEP, FAEMS

Penn State College of Medicine, Hershey, PA

Publishing \& Management

Ernest Wang • Managing Editor

Eason Cheung • Editorial Associate

Charisma Hasan • Editorial Associate

Brittany J. Dingler, MHS, PA-C • Senior Editorial Board Member, Co-Founder

Andrew Chen, MPPA • Associate Manager of Layout and Graphic Design

News \& Outreach

Isabel Anzani • Social Media \& Outreach Editor

Special Programs

Michael Beautyman • Director of Mentorship Program

Joseph Caruso • Associate Manager of Special Events

\section{General Information}

The Journal of Collegiate Emergency Medical Services (JCEMS) [ISSN 2576-3687] is the official scholarly, peer-reviewed journal of the National Collegiate Emergency Medical Services Foundation. JCEMS is published by the National Collegiate Emergency Medical Services Foundation.

Annual Subscription: Visit www.collegeems.com for information on purchasing institutional and personal annual subscriptions.

Reprints: Visit www.collegeems.com for information on purchasing reprints and single issues.

Correspondence (Editorial \& Business): Address mail to The Journal of Collegiate Emergency Medical Services, National Collegiate Emergency Medical Services Foundation, PO Box 93, West Sand Lake, NY 12196. Email: JCEMS@CollegeEMS. com

Correspondence (NCEMSF): Address mail to National Collegiate Emergency Medical Services Foundation, PO Box 93,West Sand Lake, NY 12196. Email: info@ ncemsf.org

Copyright: The Journal of Collegiate Emergency Medical Services is an open access publication. Individual authors retain copyright over their own articles. Articles are distributed under the terms of the Creative Commons Attribution 4.0 International (CC BY 4.0) License, which permits unrestricted use, distribution, and reproduction in any medium, provided the original author and source are credited. The full license is available at: https://creativecommons.org/licenses/by/4.0/. All other content in the journal is copyrighted by the National Collegiate Emergency Medical Services Foundation. All rights reserved.

Online availability: All articles published in print issues of JCEMS are available open access at https://www.collegeems.com. Additional content - not available in print issues - is also published on-line.

Disclaimers: The statements and opinions in articles or other content contained in JCEMS are solely those of the individual authors, contributors, advertisers, and sponsors, and do not represent those of JCEMS, the National Collegiate Emergency Medical Services Foundation, or any representatives, agents, or licensors. The appearance of advertisements does not represent a warranty, endorsement, or approval of the products or services advertised. JCEMS, the National Collegiate Emergency Medical Services Foundation, or any representatives, agents, or licensors make no warranties, representations, or other claims as to the accuracy or completeness of any articles or other content contained in JCEMS. JCEMS and NCEMSF disclaim responsibility for any injury to persons or property resulting from ideas, products, or other content referred to in articles or other content in JCEMS. EMS providers should always consult medical direction and local EMS protocols.

Instructions for Authors: Instructions for authors may be found in the JCEMS Guide for Authors at https://www.collegeems.com. Authors are required to abide by the latest guidelines available on-line at the time of submission.

Reviewers: JCEMS employs a double-blind peer review process for Original Research, Case Reports, and Reviews. The clinical and scientific quality of this publication relies on the rigorous and diligent reviews provided by independent reviewers with subject-matter expertise. JCEMS is grateful for all anonymous independent reviewers who dedicated their time and expertise to the collegiate EMS community. 


\title{
Creating a Protocol for Campus EMS Response to Mental Health Complaints
}

\author{
Inaya Riaz, BA, EMT; Gal Lapid, EMT; Mihir Kumar, EMT; Ravi Trivedi, BS, BA, EMT; Sulley Park, EMT
}

Keywords: collegiate-based emergency medical services; mental health | Corresponding Author and Author Affiliations: Listed at the end of this article.

$\mathrm{D}$ ue to the critical, short-term nature of emergency medical treatment, many emergency medical services (EMS) personnel currently do not have adequate resources for patients suffering from acute or chronic mental health complaints. In the United States, nearly 1 in 5 adults suffer from a mental illness within their lifetimes, suggesting the importance of regularly evaluating mental wellness during an emergency medical technician (EMT)'s assessment. ${ }^{1}$

In California, insufficient treatment among patients suffering from mental health conditions is unfortunately common. According to the 2018 California Health Care Almanac, over $50 \%$ of patients who have mental health disorders go untreated, regardless of whether or not they have insurance. ${ }^{2}$ At the college undergraduate level, it is clear that untreated mental illness is incredibly pervasive. ${ }^{3}$ College students experience unique stressors, including increased workload, interpersonal relationships, and making decisions independently. Because roughly $75 \%$ of lifetime cases of psychiatric disorders manifest by age 24 and recent years have seen a notable increase in reported mental health symptoms among college students, it is essential for universities to create and implement support systems for students' experiencing mental health crises. ${ }^{4}$ The increase in symptoms of mental health conditions and demand for mental health services also has significant implications for collegiate-based emergency medical organizations, who

Inaya Riaz, BA, EMT completed her B.A. in Medical Anthropology in 2021 and is working towards completion of a M.S. in Global Medicine, both from the University of Southern California. She served as an EMT and a board member for EMSC from 2018 through 2021. Outside of USC, she has worked in ambulance and disaster cycle positions in her community, and continues to work full time with an interdisciplinary street medicine team under the Los Angeles Department of Health Services, providing essential medical care to unhoused populations. Gal Lapid, EMT is a fourth-year student at the University of Southern California, a current candidate for a B.S. in Health Promotion and Disease Prevention and a Master of Public Health. She has served as an EMT since 2019 and a board member for EMSC since 2020. She has worked as an EMS Reserve on a Rescue Ambulance and currently works as a Public Policy intern at People Assisting The Homeless (PATH). Mihir Kumar, EMT is a fourth-year student at the University of Southern California, a current candidate for a B.S. in Human Biology and a B.A. in Economics. He was certified in the Summer of 2019 and has been a member of EMSC since the Fall of 2019. Ravi Trivedi, BS, BA, EMT, is a first-year medical student at the Western Michigan Homer Stryker MD. School of Medicine. He received a B.S. in Neuroscience and a B.A. in Economics from the University of Southern California in 2021. He was certified in the Summer of 2018 and has been a member of EMSC since the Fall of 2019. Sulley Park, EMT is a fourth-year student at the University of Southern California, a current candidate for a B.S. in Health Promotion and Disease Prevention. He was certified in the Fall of 2019 and has been a member of EMSC since the Spring of 2020. are tasked with responding to medical emergencies for their fellow classmates. Collegiate EMS programs have grown with an impressive rate over the past twenty years, yet there remains a lack of research and evidence based guidelines surrounding how we can best serve college students in need of behavioral health services. Within the Emergency Medical Services of USC (EMSC), a student-run event-based volunteer EMT program at the University of Southern California, our staff have witnessed multiple occurrences of patients self-diagnosing and self-treating severe psychiatric conditions--like depression, anxiety, and ADHD. Because we only treat our patients on a short-term stand-by basis, and typically for chief complaints unrelated to their complex mental health medical histories, there appears to be little to do to encourage them to seek long term care. Exasperating this issue is the fact that while many organizations exist to help patients with severe mental illness (SMI), low treatment rates indicate that lack of awareness and social stigma are two of the biggest barriers to treatment for these populations. ${ }^{5}$

The problems of awareness and stigma can first be witnessed and potentially addressed at the EMT level. By training first responders to not only navigate SMI calls but to also provide information on treatment options and available resources, providers can work to increase the rate of treatment for those suffering from SMIs through contact at what is often the initial step of a care cascade. In addition to this, by providing this information, EMTs would effectively initiate patient-centered care methods, which studies suggest is crucial in treating individuals with mental illness, especially for those with serious mental illnesses who have much higher dropouts and attrition rates from ongoing treatment. ${ }^{6}$

The first step in improving the EMS response towards SMI cases is to educate first responders on identifying cases where mental health plays a role in the patient's situation. Due to stigmas clouding mental health (i.e. lack of understanding by family, friends, etc., feelings of isolation and shame, reluctance to ask for help/seek treatment), it might not always be immediately clear that a call pertains to a mental health crisis. However, by forming context around the patient's situation through questions, EMTs can elucidate the mental health components to a patient's situation. In this review, we evaluate existing resources, their potential impact on and integration into EMSC and other collegiate EMS programs, barriers to treatment, and next steps for mental health integration and awareness in EMS. 


\section{The College Demographic \& SDOH}

The link between an individual's social environment and their health has long been recognized within the domains of public health and medical care systems. A mounting body of research suggests that persistent disparities in health status can be traced to largely inescapable underlying factors including race, ethnicity, age, income level, education level, sexual orientation, geographic location, interpersonal, family, and community dynamics, housing quality, social support, employment opportunities, work and/or school conditions and other factors contributing to an individual's built environment. ${ }^{7}$ These underlying factors, termed social determinants of health $(\mathrm{SDOH})$, are defined by the World Health Organization (WHO) as "conditions in which people are born, grow, live, work, and age...circumstances [which] are shaped by the distribution of money, power and resources at global, national and local levels." ${ }^{8}$ The relationship between $\mathrm{SDOH}$ and health status has become especially evident during the COVID-19 pandemic, which has exacerbated the nation's systemic inequalities and disproportionately affected racial minorities and low-income communities. ${ }^{9}$

The U.S. fee-for-service health care system has historically struggled to integrate public health and preventive services into their framework, largely because they had little incentive to do so. ${ }^{10}$ The consequences of these social determinants are starkly evident within emergency medical services (EMS), where frontline providers are tasked to treat the patient's chief complaint, but are often unable to address upstream issues of homelessness, addiction, food insecurity, racism, and more--which are some of the main predictors of a patient's health outcomes.

College environments and early adulthood bring about significant changes that impact students' SDOH. One manifestation of these changes is the disproportionately high rate of suicide. Between 2007 and 2017, the rate of death by suicide among individuals aged 15 to 24 had increased by $57 \%{ }^{11}$ As of 2018 , suicide was the second leading cause of death for the same age group. ${ }^{12}$ Similarly, rates of suicide ideation, planning, and attempt are higher among adults aged 18 to 25 compared to older adults. ${ }^{13}$ In 2015, a national survey conducted across 108 institutions found that approximately 1 in 5 undergraduate students had considered suicide, 1 in 10 had made an attempt, and nearly 1 in 5 reported self-harm. ${ }^{14,15}$ This disparity has likely been worsened by COVID-19. Many students are now living out-of-state due to stay-home orders and experience decreased access to campus therapists since licensure laws prohibit or limit practice across state borders ${ }^{16}$

While medical providers increasingly recognize the need to address $\mathrm{SDOH}$ in patient care, the process of implementation remains less clear, especially as it pertains to EMS providers, who are often limited by a lack of time and resources. There is a clear need for EMS training programs and employers to develop guidelines on the best practices to identify and address social determinants in prehospital care. ${ }^{17}$ In the interim to the creation and implementation of such guidelines, many emergency departments and some community paramedicine programs have begun to use their expertise and leadership to develop screening and referral practices to bridge the divide between emergency services and the social determinants of health. ${ }^{18}$ After a review of EMS initiatives to implement social determinants into their care framework, we defined programs as successful if their results demonstrated significant increases in post-training gains across domains related to SDOH knowledge and attitudes and/ or skills pertaining to mental health de-escalation and referral, as compared to baseline assessments. From this classification, we observed that collegiate EMS organizations who had been successful in creating programs specifically to response to mental health complaints on campus shared the following three elements: 1) standardized screening guidelines 2) integrated referral practices and 3) community partnerships. Accordingly, we recommend collegiate EMS programs to take the following measures to better integrate emergency services and social determinants of health into the acute treatment of complex mental and physical health complaints:

\section{Standardized Screening}

While clinical guidelines increasingly recommend the $\mathrm{SDOH}$ be captured and recorded, there is a lack of standardized screening tools and processes. A standardized screening process is essential to allow different players across healthcare and social service systems to communicate effectively and have a common frame of reference. Prehospital providers can implement the Question, Persuade, and Refer (QPR) Screening Tool into their secondary assessment and can use the results to inform patients' treatment plans and make referrals to community services.

\section{Our Intervention: Question, Persuade, Refer (QPR)}

To improve screening for mental illness, we recommend collegiate EMS programs incorporate gatekeeper training to improve suicide prevention, especially given that an increase in self-diagnosed and self-treated mental illnesses is likely when campuses open. ${ }^{19}$ Collegiate EMS must be prepared to provide adequate care within its scope, and gatekeeper training can empower it to do so.

Gatekeeper training is a nationally recognized suicide prevention program. In one or two hours, one learns to identify at-risk individuals and refer them to counseling and support services. Although there is no prerequisite education for the training, an ideal gatekeeper is "strategically positioned" to recognize and refer individuals. ${ }^{20}$ Collegiate EMTs are ideal candidates: not only do they have high proximity and familiarity with fellow students, but they are also likely to encounter mental health crises on calls, or to notice warning signs of mental illness during secondary assessment.

In addition, principles of gatekeeper training align with those of a well-established EMS procedure. Question, Persuade, and 
Refer (QPR) is the most widely used gatekeeper program in the United States and is recommended by the Substance Abuse and Mental Health Services Administration (SAMHSA). QPR shares procedural similarities with cardiopulmonary resuscitation (CPR). At their cores, both aim to increase a patient's chances of survival by prioritizing early recognition and intervention..$^{20}$ Although CPR does have a more demonstrable effect on patient survival, QPR education has been proven to significantly improve attitudes, subjective norms, and perceived behavioural control regarding suicide intervention. ${ }^{21}$ QPR training for EMTs can serve as an extension to their life-saving skills, allowing them to remain calm and rely on a standardized procedure when responding to mental health crises.

The University of Southern California offers micro-grant applications to recognized student organizations through the student health center's wellness program. Applying such a microgrant to the QPR training of EMTs that serve our student population invests resources back into our community to promote the long-term wellbeing of our student population and the ability of our EMTs to respond to such calls effectively. Fortunately, such funding is not limited to the University of Southern California. Most colleges and universities across the nation provide similar application-based funding to recognized student organizations through their undergraduate government bodies. At the minority of institutions that may not have such robust funding, organization-led crowd fundraising efforts through GoFundMe or alternative methods (bake sales, member dues) could also provide the necessary funding for such a program.

Limitations: The major limitations regarding QPR in EMS involve the period of time that training remains effective. Previous studies indicate that QPR training was effective in increasing immediate knowledge, attitudes, and skills to prevent suicide; however, these effects were only studied in a short-term time frame. ${ }^{22}$ Without scheduled refresher courses, the knowledge gained from QPR training can fade and cause providers to be less confident in approaching mental health emergiencies. The period of time in which training remains effective varys from provider to provider, but it has been shown that in two-year post-training follow ups, EMS providers felt significantly less confident, knowledgeable, and prepared to deal with mental health crises. ${ }^{23}$ Further research is needed on the efficacy of long-term and systemic efforts to integrate QPR into EMS. Regardless, we recommend QPR training--it is an evidencebased intervention that provides sorely needed skills to address high rates of suicides on college campuses. Furthermore, EMS on the collegiate level might be more conducive to continuous QPR refreshers than traditional EMS organizational structures. Because new students join collegiate EMS organizations every year, there is a constant need for training. By involving all crew members in this training, collegiate EMS organizations can prevent knowledge loss and provide consistent, high-quality care to the populations they serve.
Although there is a paucity of research on QPR with regard to collegiate EMS, there is evidence to support its efficacy in similar settings and populations. QPR in a college environment was effective in increasing immediate knowledge, attitudes, and skills to prevent suicide, as demonstrated through a short-term study conducted with residence advisors. ${ }^{22}$ A methodologically similar two-year study supports short and long-term results with gatekeepers who work with youth. ${ }^{23}$ Further research is needed on long-term and systemic efforts to integrate QPR. Regardless, we recommend QPR training--it is an evidencebased intervention that is theoretically a match for EMTs, and provides sorely needed skills to address high rates of suicides on college campuses.

\section{Integrated Referral Practices}

To bolster the presence of integrated referral practices during collegiate EMS calls, it is imperative that collegiate EMS programs work with their university's health departments to identify mental health resources for students of various backgrounds, ranging from free group therapy options to individual therapy and more.

\section{Our intervention: Student-Health Center Partnership}

It is important that we recognize our limited role in the long-term care of patients, as in-field stand-by EMTs. Unlike a majority of other collegiate EMS organizations, we are not involved in routine calls or patient transport. As such, the time and resources we have to provide care to patients needing longitudinal support can often be inadequate. Thus, it becomes vital to build a strong relationship with long-term mental health care resources in our community. The most accessible and advertised resource for our student population is the Engemann Student Health Center, located on the larger of our two campuses. The staff continually implement quality improvements to stretch their resources in service of our student population. Their preference is to screen and refer students, rather than offer long term care within their own facilities--a result of the immense demand for care among our student population of over 20,000. Reasonably, a student not immediately referred to an Emergency Department after leaving our care would likely be referred to Engemann. But, a gap exists in care-seeking behavior for students whose mental health concerns are not urgent enough to require immediate continued care. $^{24}$

As such, our EMS agency reached out to the student health staff at Engemann to build a partnership for direct referrals, with the general guidelines of consent and HIPAA compliance preserved in all activities. This serves to eliminate the barrier of actively needing to seek care multiple times (at least once from our EMTs, then again for continuity of care), and feeds well into a potential recipient of our QPR program. When referring students to further care, compliance improves when care is accessible, both in location and familiarity. ${ }^{23}$ For our specific resource-based intervention, we extrapolate the broad value of connecting EMS 
to continued care whenever possible. As such, we encourage other collegiate EMS programs to likewise seek longitudinal partnership with primary care and urgent care providers at their institutions' student health centers or other nearby healthcare institutions.

Limitations: At the time of publication, we have not yet received a formal response from a representative at the Engemann student health center about formally implementing a direct referral program for patients we respond to on shift experiencing a mental health crisis but we remain open to partnership and continue to explore other community-based options for referrals to continued mental health care. While EMSC already has a working relationship with a Health Promotion Specialist at Engemann who advises us about crisis de-escalation on shift and wellness practices for our student EMTs, we believe there remains a gap in the transfer of care between the immediate mental health services we provide on shift and longer-term mental health treatment offered at Engemann. Expanding our partnership with Engemann to include direct referrals for patients experiencing a mental health crisis would better address the upstream factors leading to the emergent situation. As EMSC and other collegiate EMS programs become more deeply integrated into university's emergency response systems, collaboration both within and between existing institutions is necessary to develop proactive solutions to best promote the health and safety of the students these institutions serve.

\section{Community Partnerships}

Collegiate EMS programs can assist in expanding awareness of existing resources through building partnerships with the university's mental health programs, local mental health clinics, and on-campus, student-run organizations. Once mental health resources are identified for a broad array of student populations, covering various combinations of $\mathrm{SDOH}$, collegiate EMS programs can package these resources into small infographics, brochures, etc. that may be handed out to all patients during calls. Furthermore, after the standardized screening in step 1, the EMS provider may have a better understanding of which specific

Figure 1: Our most recent card template, revised in Fall 2020 (front)

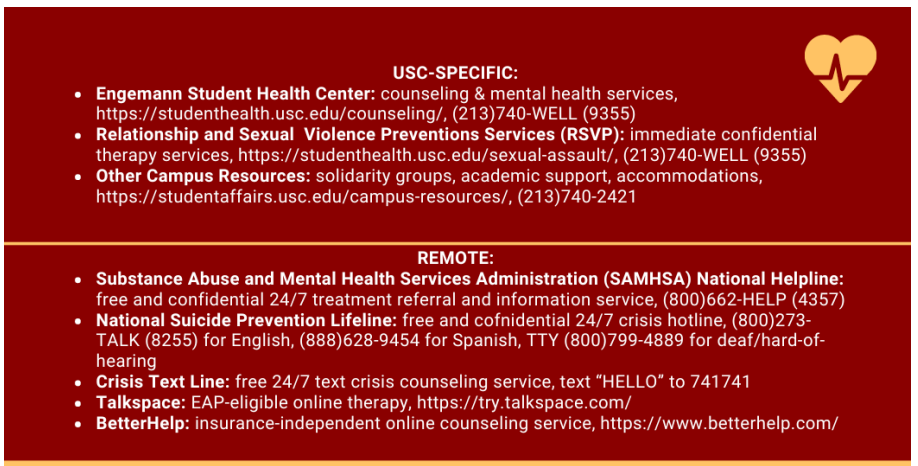

EMSC RESOURCE CARD mental ailment(s) the patient may be suffering from. At this point, the provider may be able to provide more specific, premade resources to the patient so they are able to seek treatment beyond the short-term treatment that the provider offers.

\section{Our Intervention: Patient Resource Card}

As an agency, we implemented a shift data review and classification process in the Spring of 2019 to develop evidencebased quality improvement strategies. The process involved a confidential review of shift employee records to code data by call type, frequency, partner agency dynamics, and extraordinary circumstances. A manual inductive approach was used to analyze and code the aggregated qualitative and quantitative information to identify recurring trends. This data was then used to facilitate actionable decision making in our organization, whether in continued training or mediated debriefs with staff. We rapidly noticed an alarming prevalence of mental health concerns (both chief and secondary) in our call logs. In formal post-shift debriefs, our EMTs often noted a sense of helplessness when attempting to offer further care resources to such patients, only vaguely referencing our student health center and national resources while on scene. EMTs receive very little mandated training on mental health; further, they rarely receive any training at all from employers on local mental health resources for patients. The nature of our collegiate EMS program involves high turnover, which makes changes to foundational protocol more effective than cohort training alone. After implementing a dedicated psychiatric emergencies lecture into our skills training sessions curriculum, we decided to pursue broader protocol reform to ensure a lasting impact on EMT preparedness to handle calls that are psychiatric in nature, whether emergent or not.

Thus, we added a gear item to our oxygen bags, alongside our clipboards and patient care reports: a mental wellness resource card. The card is discreet in size and includes references to campus-specific, community-specific, cost-specific, and national/remote care. Given the nature of the COVID-19 pandemic and its resulting halt of recreational events which

Figure 2: Our most recent card template, revised in Fall 2020 (back)

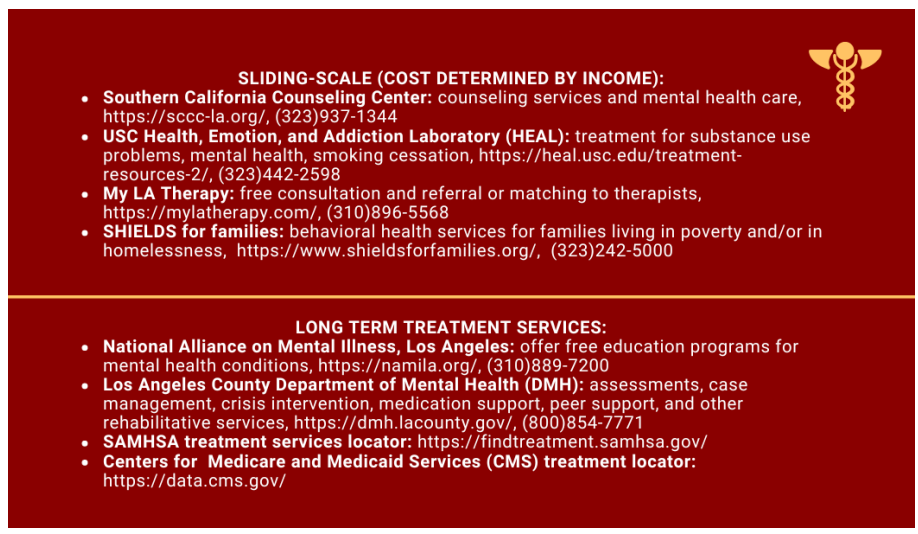


our organization traditionally staffs, we have not yet received real-time feedback from our community as to the efficacy of the resource card. The card, however, is designed to be reevaluated as necessary by EMSC's Director of Internal Relations (a fairly new role, only proposed in Spring 2019), who is responsible for staff wellness, personnel logistics, and conflict management. As the nature of internal affairs management is vastly different depending on collegiate EMS program size and resource capacity, we recommend organizations seek out a distribution of responsibility (i.e. tasking this to student volunteers versus outsourcing to credentialed professionals) best suited to their own needs.

Limitations: In order to create sustainable partnerships with local resources, we promote going beyond raising awareness to the formation of concrete and fluid partnerships. By working with the university's mental health programs and local mental health clinics, collegiate EMS programs will first identify the most important resources to build awareness about. Then, by working with on-campus, student-run organizations, collegiate EMS programs can provide brief presentations on existing resources. Furthermore, collegiate EMS programs may be able to build websites with tools (such as brief quizzes or surveys) to walk students through which mental health resource(s) may be best for them during a specific crisis. By expanding awareness of such resources through presentations and partnerships with oncampus student-run organizations and personalizing them to the student's needs with quizzes and/or surveys, collegiate EMS programs would be able to greatly demystify the daunting steps required to seek mental health resources.

\section{Conclusion}

Prehospital providers work within the community setting and are well positioned to connect medical care with social and community resources. As students themselves, collegiate EMS providers are uniquely situated to acutely understand the social factors impacting their classmates and how to compassionately refer them to relevant community services and resources. By tailoring the proposed intervention to best suit the needs of the receiving demographic, EMS agencies can best equip their staff to respond appropriately and effectively to complex calls, especially those involving mental health complaints.

\section{References}

1. Mental illness. NIMH. November 2, 2020. https://www.nimh.nih.gov/ health/statistics/mental-illness.shtml. Accessed December 19, 2020.

2. Holt W. Mental health in California. CHCF. March 28, 2018. https:// www.chcf.org/publication/2018-edition-mental-health-ca-for-too-manycare-not-there/\#: :text=Mental\%20health\%20disorders $\% 20$ are $\% 20$ among,carry\%20out\%20major\%20life\%20activities. Accessed December $21,2020$.

3. Blanco C, Okuda M, Wright $\mathrm{C}$, et al. Mental health of college students and their non-college-attending peers. Arch Gen Psychiatry. 2008;65(12):14291437. doi:10.1001/archpsyc.65.12.1429
4. Lattie EG, Lipson SK, Eisenberg D. Technology and college student mental health: challenges and opportunities. Front Psychiatry. 2019;10(246). doi: 10.3389/fpsyt.2019.00246

5. Knaak S, Mantler E, Szeto A. Mental illness-related stigma in healthcare. Healthc Manage Forum. 2017;30(2):111-116. doi:10.1177/0840470416679413

6. Pedrelli P, Nyer M, Yeung A, Zulauf C, Wilens T. College students: mental health problems and treatment considerations. Acad Psychiatry. 2015;39:503-511. https://dx.doi.org/10.1007\%2Fs40596-014-0205-9

7. Social determinants of health. USDHHS. https://health.gov/healthypeople/ objectives-and-data/social-determinants-health. Accessed December 19, 2020

8. Social determinants of health. WHO. https://www.who.int/gender-equityrights/understanding/sdh-definition/en/. Accessed December 19, 2020.

9. COVID-19: health equity considerations and racial and ethnic minority groups. CDC. July 24, 2020. https://www.cdc.gov/coronavirus/2019-ncov/ community/health-equity/race-ethnicity.html. Accessed December 19, 2020 .

10. Litteken C, Sale E. Long-term effectiveness of the Question, Persuade, Refer (QPR) suicide prevention gatekeeper training program: lessons from Missouri. Community Men Health J. 2017;54(3):282-292. https://doi. org/10.1007/s10597-017-0158-z

11. Lurie N, Fremont A. Building bridges between medical care and public health. JAMA. 2009;302(1):84-90. doi:10.1001/jama.2009.959

12. Curtin SC, Heron M. Death rates due to suicide and homicide among persons aged 10-24: United States, 2000-2017. Centers for Disease Control and Prevention. October 17, 2019. https://www.cdc.gov/nchs/products/ databriefs/db352.htm. Accessed December 13, 2020.

13. Suicide. NIMH. https://www.nimh.nih.gov/health/statistics/suicide.shtml. Accessed December 13, 2020.

14. Piscopo K, Lipari RN, Cooney J, Glasheen C. Suicidal thoughts and behavior among adults: results from the 2015 national survey on drug use and health. SAMHSA. 2016. https://www.samhsa.gov/data/sites/default/ files/NSDUH-DR-FFR3-2015/NSDUH-DR-FFR3-2015.htm. Accessed December 19, 2020.

15. Liu $\mathrm{CH}$, Stevens $\mathrm{C}$, Wong $\mathrm{SH}$, Yasui M, Chen JA. The prevalence and predictors of mental health diagnoses and suicide among U.S. college students: implications for addressing disparities in service use. Depressand Anxiety. 2018;36(1):8-17. https://doi.org/10.1002/da.22830

16. Lumpkin L. College students count on their schools for mental-health help, but now many can't get it. The Washington Post. June 7, 2020. https://www. washingtonpost.com/local/education/college-students-count-on-theirschools-for-mental-health-help-but-now-many-cant-get-it/2020/05/28/ e6f6e710-8b2b-11ea-ac8a-fe9b8088e101_story.html. Accessed December 13,2020 .

17. Axelson DJ, Stull MJ, Coates WC. Social determinants of health: a missing link in emergency medicine training. AEM Education and Training. 2017;2(1):66-68. doi:10.1002/aet2.10056

18. Andermann A. Screening for social determinants of health in clinical care: moving from the margins to the mainstream. Public Health Rev. 2018;39(1). doi:10.1186/s40985-018-0094-7

19. What is QPR? QPR Institute. https://qprinstitute.com/about-qpr. Accessed December 13, 2020.

20. Mitchell SL, Kader M, Darrow SA, Haggerty MZ, Keating NL. Evaluating 
Question, Persuade, Refer (QPR) suicide prevention training in a college setting. J Coll Student Psychotherapy. 2013;27(2):138-148. https://doi.org/1 0.1080/87568225.2013.766109

21. Aldrich RS, Wilde J, Miller E. The effectiveness of QPR suicide prevention training. Health Education Journal. 2018;77(8):964-977. doi:10.1177/0017896918786009

22. Tompkins TL, Witt J. The short-term effectiveness of a suicide prevention gatekeeper training program in a college setting with residence life advisers. J Prim Prev. 2009;30(2):131-149. https://doi.org/10.1007/s10935009-0171-2

23. Hunt J, Eisenberg D. Mental health problems and help-seeking behavior among college students. J Adolesc Health. 2009;46(1):3-10. https://doi. org/10.1016/j.jadohealth.2009.08.008

24. Dixon LB, Holoshitz Y, Nossel I. Treatment engagement of individuals experiencing mental illness: review and update [published correction appears in World Psychiatry. 2016 Jun;15(2):189]. World Psychiatry. 2016;15(1):13-20. doi:10.1002/wps.20306

Author Affiliations: From The Emergency Medical Services of the University of Southern California - in Los Angeles, CA (I.R., G.L., M.K., R.T., S.P.).

Address for Correspondence: Inaya Riaz, BA, EMT | Email: iriaz@usc.edu

Conflicts of Interest/Funding Sources: By the JCEMS Submission Declaration Form, all authors are required to disclose all potential conflicts of interest and funding sources. All authors declared that they have no conflicts of interest. All authors declared that they did not receive funding to conduct the research and/ or writing associated with this work.

Authorship Criteria: By the JCEMS Submission Declaration Form, all authors are required to attest to meeting the four ICMJE.org authorship criteria: (1) Substantial contributions to the conception or design of the work; or the acquisition, analysis, or interpretation of data for the work; AND (2) Drafting the work or revising it critically for important intellectual content; AND (3) Final approval of the version to be published; AND (4) Agreement to be accountable for all aspects of the work in ensuring that questions related to the accuracy or integrity of any part of the work are appropriately investigated and resolved.

Submission History: Received December 23, 2020; accepted for publication August 5, 2021.

Published Online: October 31, 2021

Published in Print: October 31, 2021 (Volume 4: Issue 2)

Reviewer Information: In accordance with JCEMS editorial policy, Advice and Practice manuscripts are reviewed by the JCEMS Editorial Board and, as needed, independent reviewers. JCEMS thanks the Editorial Board members and independent reviewers who contributed to the review of this work.

Copyright: ( 92021 Riaz, Lapid, Kumar, Trivedi \& Park. This is an open access article distributed under the terms of the Creative Commons Attribution 4.0 International (CC BY 4.0) License, which permits unrestricted use, distribution, and reproduction in any medium, provided the original author and source are credited. The full license is available at: https:/creativecommons.org/licenses/ by $/ 4.0 /$

Electronic Link: https://doi.org/10.30542/JCEMS.2021.04.02.01 


\title{
Establishing a Collegiate Emergency Medical Service: Methods, Motivations, and Advice
}

\author{
Ryan Ferdowsian, BA, NREMT; Tanvi Shah, BA, NREMT; Emma Finn, BA, NREMT; Aditi Chitre, NREMT; \\ Natalie Tsai, NREMT; Max Wragan; Ananya Koneti
}

Keywords: collegiate-based emergency medical services; development, funding | Corresponding Author and Author Affiliations: Listed at the end of this article.

$\mathrm{E}$ stablishing a collegiate Emergency Medical Service (EMS) facilitates faster, safer, and more accessible healthcare for all members of the college community. Our EMS team at the Claremont Colleges provides first-response basic life support to our community, as well as education and assistance with licensing for new and prospective student Emergency Medical Technicians (EMTs).

The Claremont Colleges are a consortium of 5 undergraduate and 2 graduate institutions. The undergraduate colleges share programs, courses, and regional location: they border each other across one square mile of land. All 5 undergraduate colleges share resources such as classrooms, dining halls, libraries, and social events. Those unfamiliar with the campus may arrive at unintended destinations as distinguishing one college from the next is difficult; this is due to the subtly defined borders between the schools. Additionally, access to buildings, classrooms, and residential halls by car is limited, catering towards pedestrian and golf cart access.

We created our service at the Claremont Colleges to meet the growing need for immediate medical assistance in our community. During medical emergencies, students were directed to call Campus Safety, who then could contact 911. The Claremont Colleges relied exclusively on third-party EMS responses prior to this program. However, the reliance on these outside services posed safety concerns as they encountered longer commute times and greater navigational difficulty on our unfamiliar campuses. Campus Safety personnel were often stationed on-scene or arrived within 2 minutes of an emergency; their presence at campus

Ryan Ferdowsian, BA, NREMT was an employee of the Campus Safety EMS, the Co-President of the Claremont Colleges EMS Club, and received his BA in Medical Economics from Pitzer College. Tanvi Shah, BA, NREMT was an employee of the Campus Safety EMS, was a member of the Claremont Colleges EMS Club, and received her BA in Neuroscience and Humanities: Interdisciplinary Studies in Culture from Scripps College. Emma Finn, NREMT was an employee of the Campus Safety EMS, was the President of the Claremont Colleges EMS Club and received her BA in Science Management from Claremont McKenna College. Aditi Chitre, NREMT is the Co-President of the Claremont Colleges EMS Club and is currently working to obtain her BA in Neuroscience and Philosophy from Claremont McKenna College. Natalie Tsai, NREMT was an employee of the Campus Safety EMS, is the Co-President of the Claremont Colleges EMS Club and is currently working to obtain her BA in Science Management from Scripps College. Max Wragan was a member of the Claremont Colleges EMS Club and is currently working to obtain her degree in Neuroscience at the University of Pennsylvania. Ananya Koneti is the Chair of External Programming at the Claremont Colleges EMS Club and is currently working to obtain her BA in Science Management from Claremont McKenna College. events and familiarity with the campus layout contributed to their rapid response times. In contrast, LA County Firefighters would arrive after 4 minutes and determine the need for private thirdparty EMS transport. If requested, private EMS would arrive after 9 minutes. We also learned that the multitude of unfamiliar responders impacted patient care. Students were less comfortable voicing their health wishes to non-consortium responders, which increased their risk and vulnerability in emergency situations. The public health benefits accompanying faster response times and collegiate-affiliated emergency services has been cited by experts in the field as well as EMS divisions at institutions such as The University of San Francisco. ${ }^{1,2}$ To address these community health concerns, an internal EMS was developed.

Accommodating the needs of our community, the Campus Safety Emergency Medical Service contributes to student wellness by employing student-EMTs. These undergraduate healthcare professionals can provide immediate care by rapidly responding to on-campus medical emergencies, professionally advocate for patients, and monitor them for symptoms of deterioration should they refuse hospital transportation.

Our service was modeled after other existing collegiate EMS programs. We partnered with UCLA, USC, LMU, Dayton, Chapman, Cal State San Bernardino, Cal State LA, Cal State Long Beach, Cal State Fullerton, and Colorado College. These schools have helped guide the assembly of a student-EMS program with their history, resources, and experience. The service was initially set to launch in the spring semester of 2020 as an extension of Campus Safety. The program was designed under the guidance of Campus Safety in order to best adapt the service to the wider community.

This article outlines the process of establishing a collegiate EMS (Figure 1) using our experience at the Claremont Colleges as a case study for prospective collegiate EMS agencies.

\section{Phases}

Phase 1: Ensure Necessity and Interest in the Program

We discovered the community's interest in and need for a studentemployed EMS by conducting a noncomprehensive survey of the student bodies from the 7 Claremont Colleges (Appendix 
Figure 1: Flowchart detailing the sequence of steps in establishing a collegiate EMS program

\section{PHASES OF DEVELOPMENT}
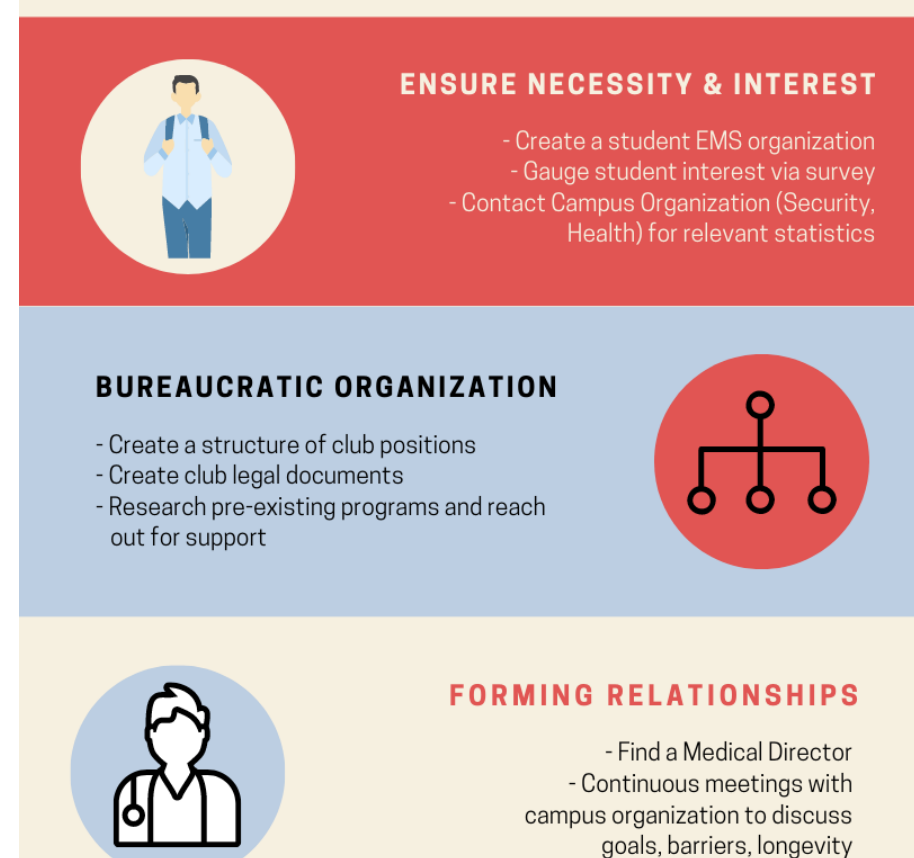

FORMING RELATIONSHIPS

- Find a Medical Director

- Continuous meetings with campus organization to discuss goals, barriers, longevity

\section{DOCUMENTATION}

- Create a potential supplies list - Draft medical protocols using preexisting templates
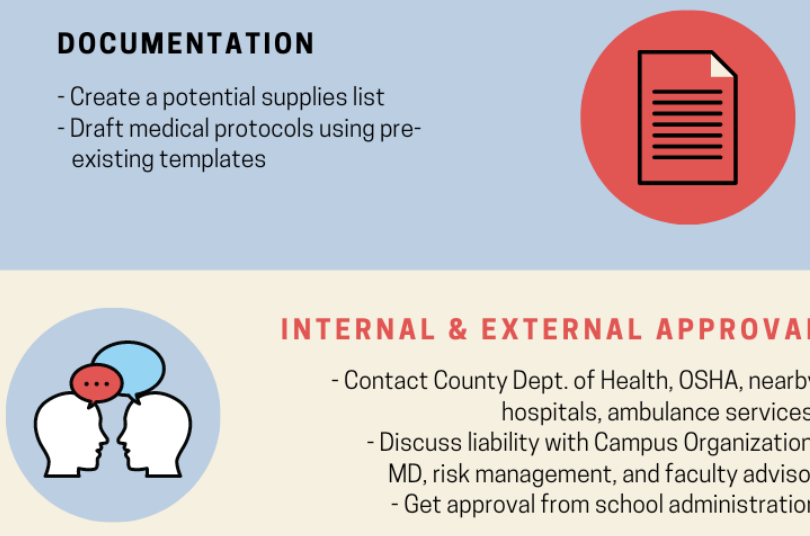

INTERNAL \& EXTERNAL APPROVAL

- Contact County Dept. of Health, OSHA, nearby hospitals, ambulance services - Discuss liability with Campus Organization, $\mathrm{MD}$, risk management, and faculty advisor - Get approval from school administration

\section{FINISHING TOUCHES}

- Finalize legal documents

- Develop partnership between Campus Service \& Third Party EMS

- Search for additional funding options

- Consider implementing a QI program
C). Recognizing the gap in medical care on campus, students advocated for improved emergency medical services. Students mentioned that they would benefit from adjustments to the current system. These include extended medical services (e.g. hours and staffing) in addition to Student Health Services, and supplementary resources for physical and mental-illness related medical resources on-campus.

Although the survey results presented an apparent need for a campus-based EMS agency, the Claremont Colleges Emergency Medical Systems team recognizes potential limitations in the study's fidelity. The survey, which was distributed to the student body via Facebook and email, did not collect demographic data. Out of a total population of 8,500 undergraduate and graduate students, our survey received between 36 and 42 respondents. In the future, prospective EMS agencies should consider utilizing a numerical scale questionnaire to assess student interest for a campus-based EMS agency. Additionally, they could more effectively inform students of the survey by utilizing students' preferred social media platforms: Instagram, Twitter, and Snapchat. ${ }^{3}$ By focusing on a quantitative and mass-circulated study, other prospective collegiate EMS agencies may be able to justify establishing the agency through more empirically-based evidence.

Nonetheless, following the culmination of our survey, we learned of further student input from anecdotal data. Students recorded a preference for disclosing personal information to a peer when in crisis and would be more likely to reach out to them as opposed to a third party for financial reasons. It became apparent that students preferred to be evaluated by a peer to determine whether they needed higher levels of care (i.e. transportation to a hospital) through conversations at events the EMS club tabled at, including campus club fairs and the EMS club's own outreach programs. Following a series of additional surveys and conversations with the student bodies, our EMS program was established to accommodate the expressed needs of our community.

After recognizing our student body's interest for a peer-driven EMS, a team of students mobilized to both obtain EMT-B licensing and train future student-EMTs in order to ensure there would be employable students when the service began. The team began researching the feasibility of a student-EMS program on campus. This involved analyzing statistical data, protocols, and operating procedures generated by established programs. Furthermore, the process of initiating a collegiate EMS was outlined during conversations with the National Collegiate Emergency Medical Foundation and the University of Dayton's EMS. After extensive research, the team ultimately decided to model the service after Colorado College's EMS since our connection with them granted us access to their resources and protocols. Furthermore, the structure and student population between the two schools was similar, making it a natural fit.

\section{Phase 2: Organization}

We established ourselves as a club open to all students of the 
Figure 2: Timeline of events in gaining EMT-Basic Certification. Additional steps may be required depending on location

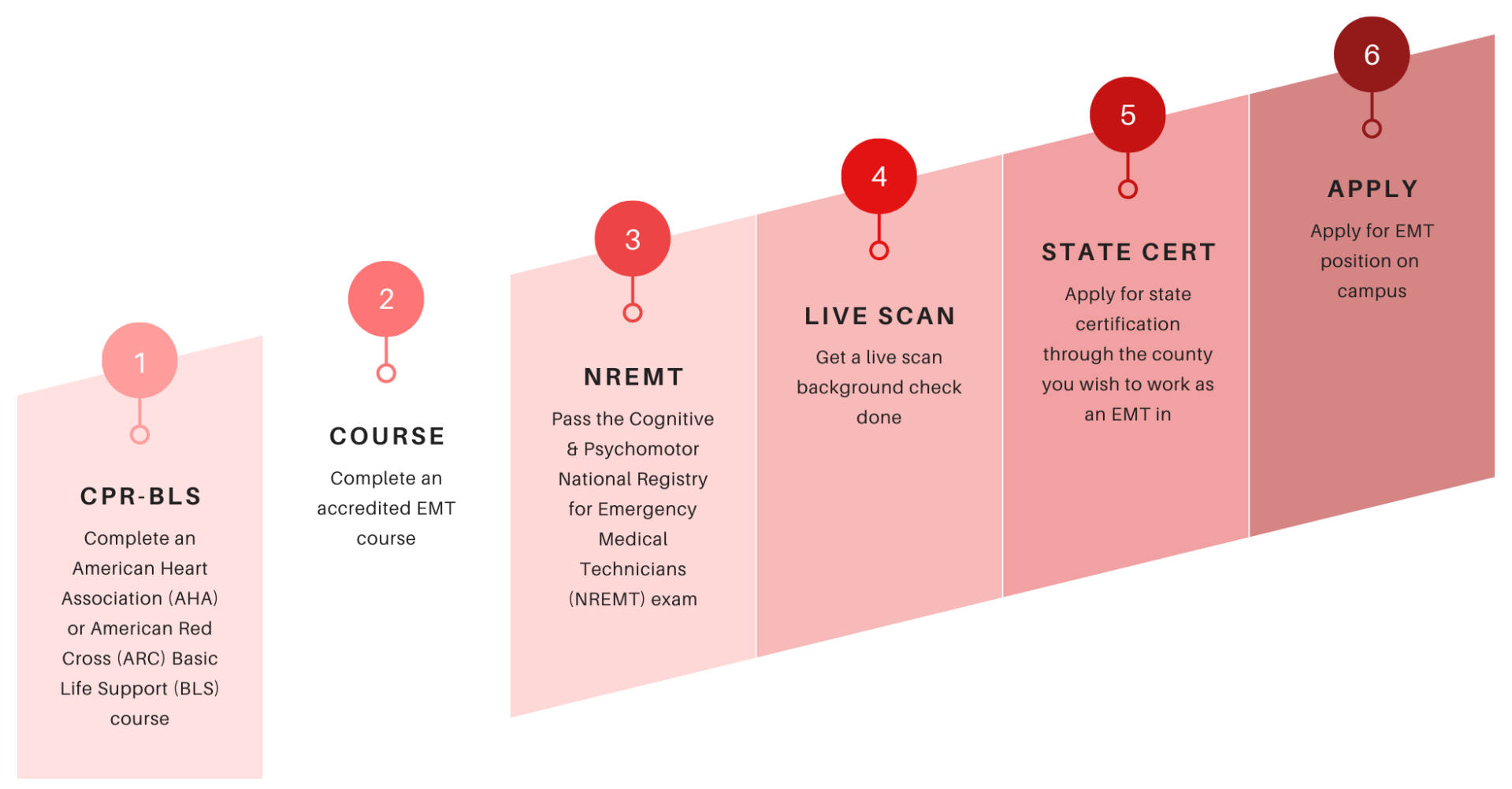

Claremont Colleges to effectively organize our interested members. Creating officer positions within our club allowed us to delegate tasks for the documentation of our mission, constitution and bylaws. As an established club, we conducted research and communicated with experts in emergency medicine by reaching out to other collegiate EMS organizations. Our team then sought out a partnership with a local EMT training school. This partnership facilitated club member certifications by creating a streamlined and consistent training method for them (Figure 2).

We were faced with the decision to either initiate our service through Campus Safety or Student Health Services (SHS), both of which serve all 7 of the Claremont Colleges. Because Campus Safety was a 24-hour service that responded to emergent medical needs, whereas SHS operated during standard clinical hours, we concluded that Campus Safety should house our service.

\section{Phase 3: Forming Relationships}

Our club then began laying the groundwork to establish our EMS. Members of our club reached out to medical directors in our area, which proved to be difficult. We searched for a medical director by asking physicians from nearby Emergency Departments and connecting with others through LinkedIn. After contacting over eighty emergency medicine doctors, we found an alumnus who became our service's medical director. Because alumni have geographical and sentimental ties to their colleges, we recommend networking with them first when searching for a medical director.

\section{Phase 4: Documentation}

After onboarding a medical director, we created a supplies list (with corresponding projected costs) and formed our medical protocols. These protocols were based on the Los Angeles County Emergency Medical Services Agency (EMSA) recommended protocols. The EMSA protocol template informed our charting layout, operating procedures, and service structure. Prior to final approval, the supplies list and protocols were reviewed by our medical director.

Other schools in the Los Angeles area aided us in drafting our protocols by providing their knowledge of local laws regarding EMS. Therefore, we recommend nascent collegiate EMS teams to contact local hospital emergency departments and nearby collegiate medical services for guidance in the development of their protocols and overall program.

\section{Phase 5: Funding and Approval}

After documenting paperwork and establishing connections for our program, our team obtained funding as well as authorizations from internal and external organizations.

On campus, we continued communicating with Campus Safety and our medical director while we began discussions with risk management. These talks revealed that insurance, liability, and funding would pose barriers to our service's establishment. While 
our medical director requested full indemnity for his services, our risk management official explained that it would not be feasible for the Colleges to assume that level of risk by themselves. During these discussions, we served as an intermediary between the two parties and emphasized the importance of modeling our service after similar EMS agencies. Furthermore, we reached out to partner collegiate EMS agencies regarding model legal relationships between risk management and medical directors. With this information, our risk management team was then able to begin formulating the structure of our legal documents. Our efforts also inspired the risk management team to contact other risk managers of local schools for questions and guidance regarding collegiate EMS insurance coverage. The risk management team utilized this information to draft legal documents that provided our studentEMTs with malpractice insurance and mitigated the liability placed on our medical director.

During this time, we also learned that student clubs organizations may not have the funds to support such an expensive program and as such forming partnerships with established community organizations may provide essential resources for the service. We required funds for our start-up costs (e.g. radios, medical vehicle) and service costs (e.g. medical supplies). Initially, our team reached out to on-campus organizations for financial assistance and partnerships. The team first looked to form a relationship with the Keck Graduate Institute School of Medicine; they could provide a source of medical supplies for the Campus Safety EMS. As the medical school was still in its early stages of initiation, the
Keck administrators mentioned that they eagerly anticipated a future partnership but would not be able to provide any financial assistance or supply exchanges until the school was established. The team also applied for funding through the Claremont Colleges clubs organization. During the presentation of our ideas and vision, we received overwhelming support for the Campus Safety EMS. However, as we required significantly more than what their budget could accommodate, we could not secure any funding. Instead, through discussions with Campus Safety, we learned that they could support our service costs but would require external financial resources to cover the start-up costs. These start-up costs were later covered by the Student Dean's Council (comprised of the respective Dean of Students from each Claremont College) who were recommended to financially support our program by Campus Safety. From these experiences, our financial team learned that a collegiate EMS should first search for funding from entities that they are already actively working with. Even if these entities do not have the funds themselves, they may recommend additional entities to contact; they will also advocate for the collegiate EMS agency.

Another barrier we encountered was obtaining official approval for the service from the Student Dean's Council (SDC). We had contacted the SDC on many occasions to formally present our program for their board. We understood that the formal proposal would be followed by an official vote for or against the program's establishment. Our efforts, however, failed to gain traction as we were denied an audience with them. We then had team

Figure 3: Geographical distribution of NCEMSF groups across the United States

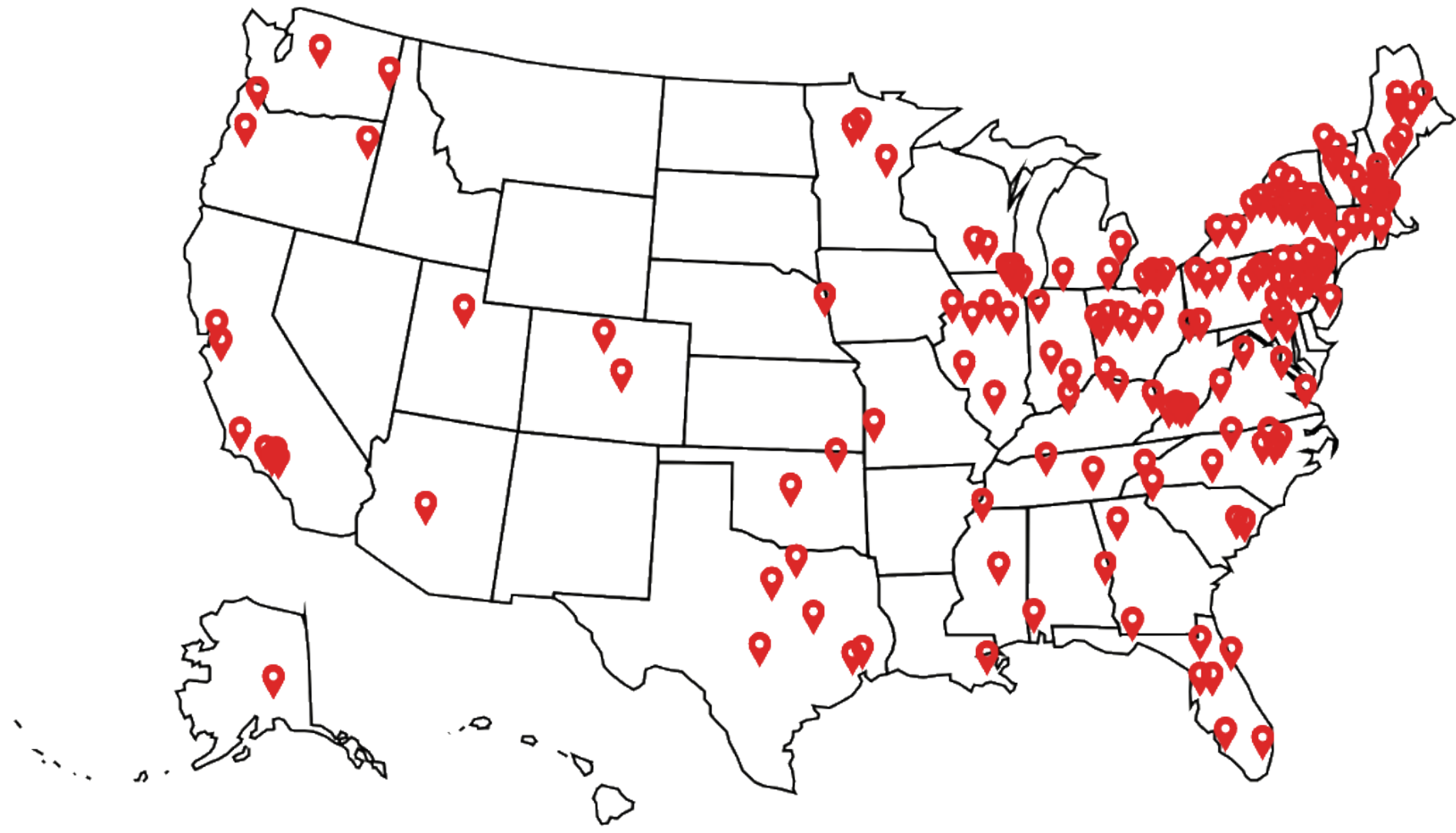

JCEMS · Volume $4 \cdot$ Issue $2 \cdot$ October 2021 
members from each Claremont College contact their respective Dean of Students and request individual meetings. During these discussions, our team members answered questions and clarified our program goals and vision. We believe that these meetings were instrumental to our eventual approval as they allowed the program to gain support from individual administrators before it was formally presented to them. Through these discussions, individual administrators also informed us that the SDC would be much more receptive to hearing the program's proposal if it were presented by an established campus organization such as Campus Safety. After explaining our findings to Campus Safety, they reached out to the SDC and were able to set up a formal meeting for the program's proposal. Campus Safety's proposal resulted in the official approval of our program: we were established as a division within Campus Safety. Through these experiences, we learned that a collegiate EMS may benefit from contacting administrators individually and from asking a more established organization to present the program on behalf of the student group. These two actions improve administrators' perceptions of the program before the formal proposal and legitimizes the program, making it more palatable for a college's administrators.

Concurrently, we obtained recommendations from external organizations like Occupational Safety \& Health Administration and the Los Angeles Department of Health. During this phase, we also recommend establishing connections with local hospitals and ambulance services for region specific advice and additional support.

\section{Phase 6: Finishing Touches}

Having secured the resources and established relationships onand off-campus, we prepared to launch the pilot phase of our program. We explored different charting systems: both paper and electronic. After verifying our training procedures with Campus Safety, an application to work on the service was created. The position was available to all Claremont College students, six of which were eventually chosen to join the team. We decided that during the pilot phase, student-EMTs would work during peak hours and respond to calls in conjunction with Campus Safety. Due to the COVID-19 pandemic, the pilot program had to be postponed. When circumstances allow our service to launch, our goal is to expand its size by adding more certified student-EMTs who can meet our campus' need for immediate medical attention.

Once the program begins, we have many goals to continually improve the Campus Safety EMS. We plan to gather data on the type of emergencies occurring (e.g. demographic, call location, call response time, severity), which will help us adapt our service to meet the specific needs of the population we serve. Additionally, the pilot phase serves to increase awareness about the campus service before the complete program is instituted.

As we continue to grow, we hope to implement a Quality Improvement program by hiring students from either of the Claremont graduate colleges, search for additional funding, and establish collaborative partnerships with county fire departments through student ride-alongs.

\section{Conclusion}

Throughout the process of creating our collegiate EMS, we realized that first-aid response and emergency medical training are often confined to the pre-health student community. The relatively small size of this community limits the number of individuals (i.e. first responders) that can engage in life-saving interventions during a medical emergency. Since collegiate EMS organizations should address a multifaceted student need, we believe that they should foster student education on emergency preparedness in addition to their primary concerns to guide interested students through the certification process.

As detailed in phase 2, our organization sought an EMS education partnership with a local EMT training facility. This partnership allowed our club members to gain EMT certifications at a subsidized rate as well as provide members with a streamlined process in attaining their certification. Colleges intending to start a collegiate on-campus service should consider fostering a relationship with a local EMT training facility. Such a partnership will make certification more accessible for students and therefore maximize the number of students with an EMT certification. Having a large group of students certified will not only help the service grow, but will also give traction to the service when gaining approval from administrative committees.

Colleges and universities seeking to implement a collegiate service on their campus can best benefit from this guide by extracting general steps from each phase outlined as summarized by Figure 1. They must also keep in mind that the process is not as clear cut as is shown in the separate phases. Certainly, some steps had to be completed before moving on to the following phase, such as securing a Medical Director and acquiring the proper documentation. Still, most tasks overlapped with each other nonlinearly, as very few of them could be quickly checked off a list in stepwise fashion. Furthermore, institution format and student population size should be taken into account; for example, larger schools may face more barriers and requirements throughout the process. For us, a unique difficulty posed by the consortium style of the Claremont Colleges was securing approval from each of the 5 colleges' administrations. Overall, flexibility and adaptation will serve other institutions well when using our experience as a guide to establish their own service.

This article addresses the detailed steps necessary to establish a collegiate EMS program. Our team at the Claremont Colleges worked tirelessly with our Dean of Students, Student Health Services, and the Campus Safety department to bring this idea to fruition, but it would not have been possible without the guidance of established organizations from other institutions. This piece is intended to provide insight into the establishment of collegiate EMS programs and serve as a comprehensive guide of important considerations. Our team at The Claremont Colleges hopes that 
this guide will facilitate your experience in creating a collegiate EMS program, and that you will use it to further emergency medical knowledge within your own community.

If you have any questions/comments pertaining to the article or would like personalized advice for establishing your collegiate EMS, please contact us at ClaremontCollegesEMS@gmail.com

\section{Authors' Remark}

Of note, 4 of our 7 authors (Ryan Ferdowsian, Emma Finn, Tanvi Shah, and Natalie Tsai) were also inaugural employees of our Campus Safety EMS. We decided to begin writing this manuscript shortly after realizing that the service would be postponed due to the COVID-19 pandemic. As members of the service, one of our primary goals was to improve community health and wellbeing. We found that this project allowed us to indirectly achieve our goal by providing resources other communities could use to similarly improve the health and wellbeing of their members. In conclusion, we urge you to be resilient when faced with unexpected challenges by searching for alternative methods to serve your community and those surrounding.

\section{Acknowledgements}

We would like to extend our gratitude to The Assistant Vice President of Campus Safety at the Claremont Colleges whose support, advocacy, and mentorship were instrumental in the creation of our service. Furthermore, we would like to recognize our medical director for his dedication to our cause and guidance in the creation of our medical protocols.

\section{References}

1. Fisher J, Ray A, Savett SC, Milliron ME, Koenig GJ. Collegiate-based emergency medical services (EMS): a survey of EMS systems on college campuses. Prehosp Disaster Med. 2006;21(2):91-66. doi: 10.1017/ s1049023x00003411. PMID: 16770998.

2. Struve OA. Design and implementation of a sustainable, university-based, emergency medical response service. Doctor of Nursing Practice (DNP) Projects. 2014;26. https://repository.usfca.edu/dnp/26

3. Fraccastoro KA, Moss G, Flosi A, Karani K. Disseminating information to college students in a complex media environment. Business Education Innovation Journal. 2020;12(1):49-53.

\section{Supplementary Materials}

Appendix A: Professionalism (Available Online)

Appendix B: Longevity Practices (Available Online)

Appendix C: Student Survey Questionnaire (Available Online)

Author Affiliations: From Campus Safety EMS; Claremont Colleges EMS Club; Pitzer College - in Claremont, CA (R.F.). From Campus Safety EMS; Claremont Colleges EMS Club; Scripps College - in Claremont, CA (T.S.). From Campus Safety EMS; Claremont Colleges EMS Club; Claremont McKenna College - in Claremont, CA (E.F.). From Claremont Colleges EMS Club; Claremont McKenna College - in Claremont, CA (A.C., A.K.). From Claremont Colleges EMS Club; Scripps College - in Claremont, CA (N.T.). From Claremont Colleges EMS Club in Claremont, CA; University of Pennsylvania - in Philadelphia, PA (M.W.).

Address for Correspondence: Ryan Ferdowsian, BA, NREMT | Email: RFerdow@students.pitzer.edu | Phone: 650-704-6639

Conflicts of Interest/Funding Sources: By the JCEMS Submission Declaration Form, all authors are required to disclose all potential conflicts of interest and funding sources. All authors declared that they have no conflicts of interest. All authors declared that they did not receive funding to conduct the research and/or writing associated with this work.

Authorship Criteria: By the JCEMS Submission Declaration Form, all authors are required to attest to meeting the four ICMJE.org authorship criteria: (1) Substantial contributions to the conception or design of the work; or the acquisition, analysis, or interpretation of data for the work; AND (2) Drafting the work or revising it critically for important intellectual content; AND (3) Final approval of the version to be published; AND (4) Agreement to be accountable for all aspects of the work in ensuring that questions related to the accuracy or integrity of any part of the work are appropriately investigated and resolved.

Submission History: Received December January 24, 2021; accepted for publication July 2, 2021.

Published Online: October 31, 2021

Published in Print: October 31, 2021 (Volume 4: Issue 2)

Reviewer Information: In accordance with JCEMS editorial policy, Advice and Practice manuscripts are reviewed by the JCEMS Editorial Board and, as needed, independent reviewers. JCEMS thanks the Editorial Board members and independent reviewers who contributed to the review of this work.

Copyright: (c) 2021 Ferdowsian, Shah, Finn, Chitre, Tsai, Wragan \& Koneti. This is an open access article distributed under the terms of the Creative Commons Attribution 4.0 International (CC BY 4.0) License, which permits unrestricted use, distribution, and reproduction in any medium, provided the original author and source are credited. The full license is available at: https://creativecommons.org/ licenses/by/4.0/

Electronic Link: https://doi.org/10.30542/JCEMS.2021.04.02.02 


\title{
Evaluating the Content and Quality of Emergency Medical Services Oral Patient Handoff Reports
}

\author{
Matthew D. Monaco, MD; Jordan J. Grove, MD; Joshua Beckedorf, MD; Walter A. Schrading, MD, FACEP, \\ FAWM
}

\begin{abstract}
Background: Annually millions of patients are brought to Emergency Departments (ED) across the US by air and ground ambulance. There exists no universal criterion for the patient data to be presented from Emergency Medical Services (EMS) to ED personnel. Prior studies show gaps in patient data during handoffs. Objectives: This study seeks to ascertain what patient data is orally reported by EMS to ED personnel. Methods: This was a prospective observational study using a convenience sample of EMS to ED transfer events documented by trained observers. Oral handoffs were evaluated for the presence of selected patient data metrics, interruptions, and whether additional data was obtained through follow-up questions. Results: In total, 102 handoffs from EMS to ED personnel were observed; 76 handoffs were medical and 26 handoffs were traumas. Chief complaints and history of present illness (HPI) were reported most frequently (94\% and 84\% respectively). Vital signs were presented initially during $44 \%$ of cases. Following the initial patient presentation, vital signs were the most requested data on follow-up questioning by receiving medical personnel (15\% of cases). Trauma patients had their physical exam, assessment, and interventions reported more frequently than medical patients but vital signs were reported slightly less so. Conclusions: The frequent absence of patient data metrics like vital signs represents possible limitations in the comprehensiveness of handoffs received by ED personnel from EMS. There appear to be opportunities to improve communication of certain key physical exam findings like vital signs.
\end{abstract}

Keywords: emergency department, handoff, communication, collegiatebased emergency medical services

Corresponding Author and Author Affiliations: Listed at the end of this article.
$\mathrm{I}$ $\mathrm{n}$ the United States in 2016, 22 million patients representing $15.8 \%$ of all Emergency Department (ED) patients arrived via ambulance. ${ }^{1}$ This proportion was greater for patients 75 years and older for whom $40.7 \%$ arrived at the ED via ambulance. ${ }^{1}$ Communication between prehospital providers (EMTs and paramedics) to ED staff is crucial in improving patient safety and reducing medicolegal risk. ${ }^{2}$

Walter Schrading, MD, FACEP, FAWM is an Associate Professor of Emergency Medicine at the University of Alabama at Birmingham. He has been teaching emergency medicine for over 30 years. He is the author of over 30 publications. As the Director of the Office of Wilderness Medicine, he teaches at numerous wilderness medicine courses and runs a specialty track in wild med for EM residents. He has been actively involved in mentoring numerous medical student research projects in emergency medicine. Joshua Beckedorf, MD is a graduating third year resident and upcoming clinical instructor of Emergency Medicine at the University of Alabama at Birmingham. His interests include Wilderness Medicine and he helps teach wilderness medicine courses to medical students and other healthcare personnel. Matthew D Monaco, MD is a prior EMT-B who volunteered providing medical aid and serving as the president of his college's all student run Rensselaer Polytechnic Institute Ambulance. He served for two years after college in the United States Navy as a Surface Warfare Officer. He has recently graduated the University of Alabama School of Medicine and is an incoming emergency medicine intern physician at Naval Medical Center Portsmouth in Portsmouth, VA. Jordan J Grove, MD has recently graduated the University of Alabama School of Medicine and is an incoming emergency medicine intern physician at San Antonio Military Medical Center. Prior to medical school he served as an officer and instructor pilot in the USAF since 2010.
The American College of Emergency Physicians and The National Highway Traffic Safety Administration (NHTSA) provides recommendations for information to be discussed during patient handoffs which include but are not limited to the chief complaint, vital signs, physical exam, and interventions performed by EMS. ${ }^{2,3}$

The success rate of these recommendations was examined in a previous pilot study in which 105 EMS personnel were surveyed on the EMS to ED hand-off with only $42 \%$ having reported receiving standardized training on the hand-off with $35 \%$ concluding that their typical hand-offs are complete. ${ }^{4}$ The above-referenced source illustrates a lack of standardization across disciplines that lead to a disparity of data reported.

Several studies have consistently shown the incompleteness of EMS to ED hand-offs. One study of critically ill patient handoffs from prehospital providers to ED staff found that less than $60 \%$ of handoffs included a complete set of patient vitals. ${ }^{5}$ Another study found that complete vital signs were given in only $53 \%$ of pediatric resuscitation cases in an emergency room and that these handoffs were further complicated by frequent interruptions and redundant questioning between EMS and ED personnel. ${ }^{6}$ In another study that observed over 600 medical hand-offs and 400 trauma hand- 
offs, it was found that the most frequently transferred data during hand-offs was events (99\% trauma, 98.1\% medical) and problems (99\% trauma, 97.5\% medical) while less frequently reported were patient allergies (around 70\%) and vital signs (70-85\% of cases). ${ }^{7}$

Previous studies have focused only on critically ill patients., ${ }^{5,6}$ One of the few studies to date that has included a large number of handoffs and medical and trauma patients was conducted over 10 years ago, between 2010-2011. ${ }^{7}$ With a limited number of studies conducted in this area, especially ones considering non-critically ill patients and involving a vital safety aspect of patient care, this area deserves further study. We hypothesized that certain patient metrics are not being transferred in a substantial number of cases.

\section{Methods}

\section{Study design}

We performed a prospective observational study of handoffs from prehospital providers to ED staff using a convenience sample.

\section{Study setting}

Research was conducted on the campus of a large city-based university emergency department with a level I trauma center which sees over 180,000 patients per year. Data were collected in each of the ED's primary receiving pods 2, 3, 4, and 5 (Trauma Bay). Data was collected from late April through June 2019. A total of 102 handoffs were observed during this time. This was a convenience sample that occurred when trained observers were available and in the department from 0900-2000.

\section{Participants/patients}

This study was reviewed by the Institutional Review Board and applied for and received a designation as Not Human Subjects Research. Patient handoffs were included in the study if patients were at least 18 years of age, not pregnant, and not incarcerated. EMS crews observed were both public and private ambulance services operating in the local area. Both air and ground EMS crews were observed. As above, patients were selected consecutively as part of a convenience sample

\section{Data collection}

Data collection was performed by an experienced emergency medicine resident and two medical students. Medical students were trained by a board-certified emergency physician. Training consisted of covering patient flow in the ED, data to be collected on the data collection sheets, and trial collections in the ED. Students were observed collecting data for accuracy several times before being allowed to independently collect. This was done by having the student and preceptor collect data together on the same case and then comparing results for accuracy.

Data were recorded on standardized data collection forms on which observers circled yes or no as to whether specific data metrics were discussed during patient handoffs from EMS to ED personnel. In this study, a handoff constituted the ongoing and dynamic transfer of patient care as EMS arrives in the ED up until the time they physically leave the patient.

During this study, the observers would await the patient's arrival

Figure 1: Most common paths a patient will take as they are brought in by ambulance to the UED

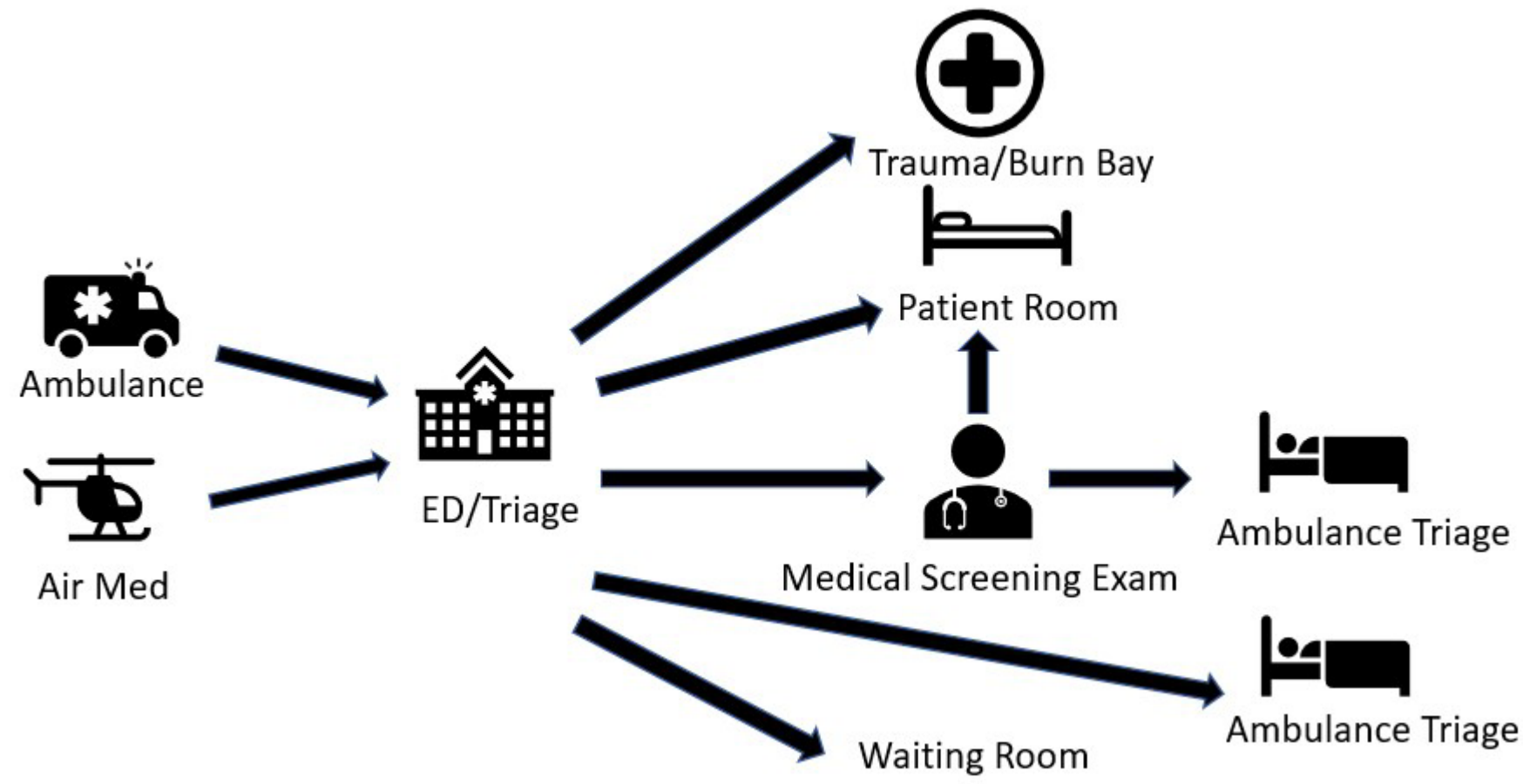

JCEMS · Volume 4 · Issue $2 \cdot$ October 2021 
via ambulance or helicopter. Once the patient had arrived the trained personnel would observe and record the handoffs. A single handoff included all oral reports EMS made on behalf of that individual patient to ED personnel including triage nurses, ED residents/attendings, trauma residents/attendings, and ED nurses. Data transferred during radio transmissions were not included.

Data metrics measured were age, gender, chief complaint, history of present illness (HPI), past/current medical and surgical history, allergies, medications, vital signs, physical exam, EMS Assessment, and EMS interventions performed and or EMS's plan. No identifying patient information was recorded at any time.

Additionally, observers recorded if at any time during a patient handoff 1) the report was interrupted, 2) ED personnel asked for data that was already presented, and 3) for each criterion that was not initially presented if the ED personnel asked for it and if EMS knew the answer.

\section{Results}

A total of 102 patient handoffs between prehospital providers and ED Staff were observed and recorded. 26 were recorded in the trauma and burn bays. Six handoffs were made by Basic Life Support (BLS) crews, 85 by Advanced Life Support (ALS) crews, and 11 by air medical crews.

Figure 2 summarizes the percentage of encounters during which a certain data point was transferred or not. Age and gender were transferred in $19 \%$ and $40 \%$ of cases respectively. Chief complaint and HPI were transferred most often at $94 \%$ and $84 \%$ percent of the time. Historical data metrics including past medical and surgical history (42\%), medications (9\%), and allergies (7\%) were all transferred less often than present illness data and objective and interpretive data.

Objective data including vital signs and physical exams were reported $44 \%$ and $56 \%$ percent of the time. EMS crews provided an assessment of the patient in $44 \%$ of cases. Plan and interventions were likewise reported in $44 \%$ of cases. EMS crews were recorded as having been interrupted during their handoffs $20 \%$ of the time. ED personnel asked EMS crews to repeat information they had already verbally reported $9 \%$ of the time.

As shown in Table 1, following EMS's initial oral reports to ED personnel the most frequently asked questions on follow-up were about vital signs and past medical or surgical history. In 15 of the cases observed EMS crews were asked for the patient's vital signs. Of those 15 cases, EMS crews were able to provide an answer $100 \%$ of the time.

Figure 3 shows a comparison of trauma $(n=26)$ vs medical $(n=76)$ EMS oral reports. The largest discrepancies exist in the rate at which age and gender were reported by EMS crews. Medical patients had their age and gender orally reported $9 \%$ and $26 \%$ of the time while trauma patients had their age and gender reported $46 \%$ and $81 \%$ of the time. Another large discrepancy exists in the rate of past medical and surgical histories provided for patients as EMS crews reported this for medical patients $51 \%$ of the time and for trauma patients $15 \%$ of the time. Physical exam and interventions performed showed crews with trauma patients more often reporting those categories in their oral reports.

\section{Discussion}

The handoff of patients from EMS to ED personnel often occurs

Figure 2: Percentage of time data metrics were transferred by EMS crews during oral handoff reports to the ED

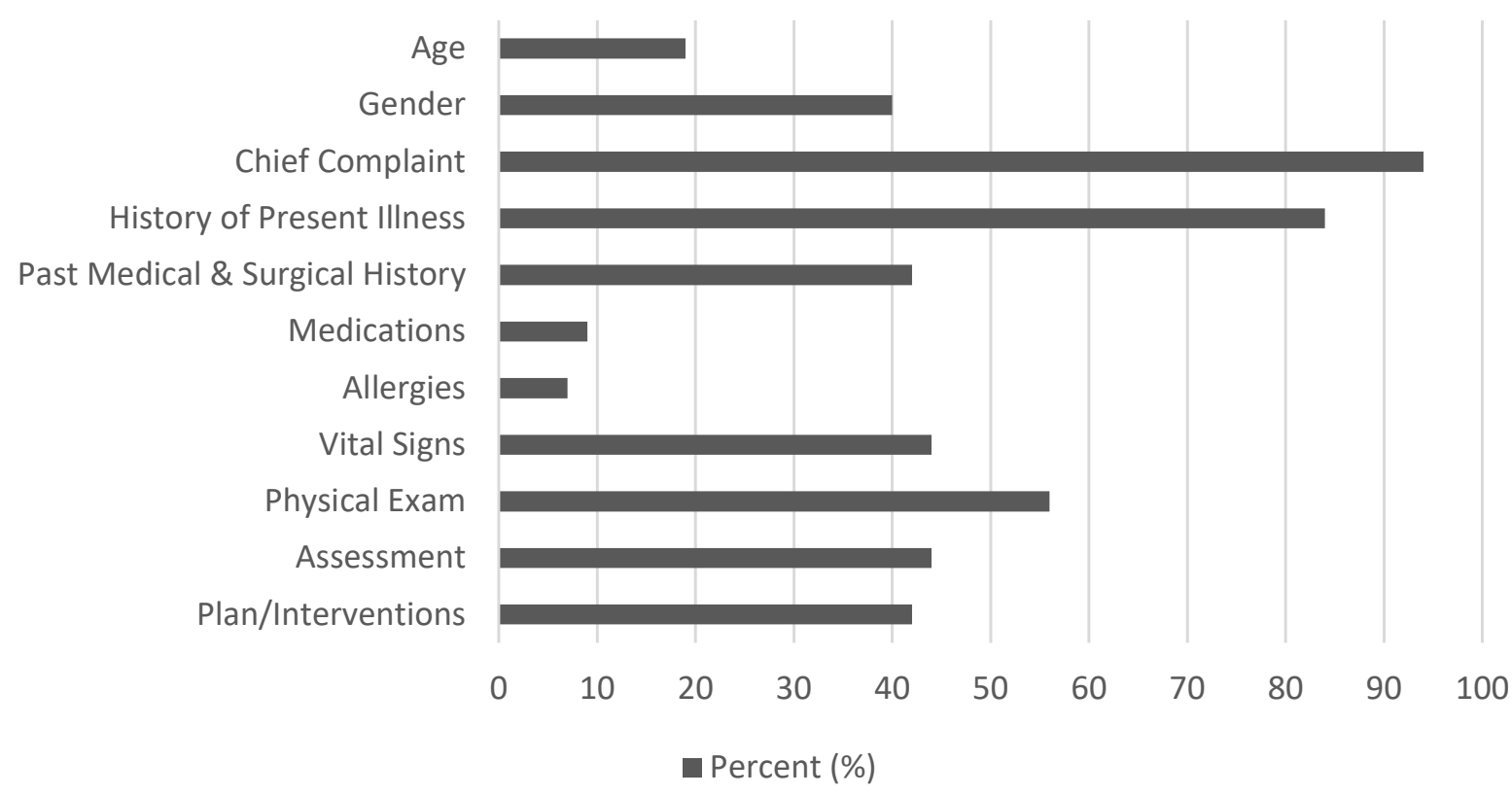


Table 1: Number of times EMS was asked a question about a data point that was not transferred and the number of times they knew the answer

\section{Times Asked Answered/(\%)}

\begin{tabular}{lcc}
\hline Age & 1 & $1(100 \%)$ \\
Gender & 0 & $0(\mathrm{NA})$ \\
Chief Complaint & 1 & $1(100 \%)$ \\
History of Present Illness & 5 & $5(100 \%)$ \\
Past Medical \& Surgical & 13 & $13(100 \%)$ \\
History & 8 & $6(75 \%)$ \\
Medications & 5 & $5(100 \%)$ \\
Allergies & 15 & $15(100 \%)$ \\
Vital Signs & 9 & $9(100 \%)$ \\
Physical Exam & 6 & $6(100 \%)$ \\
Assessment & 9 & $9(100 \%)$ \\
\hline Plan/Interventions &
\end{tabular}

in a dynamic and ever-changing environment. While patient data is often transferred electronically or in written format, oral reports represent the human-to-human transfer of a patient's care from provider to provider. The Joint Commission reported that in 2014 80\% of serious medical errors involved miscommunication between providers during patient transfers of care/handoffs leading to delays in treatment, inappropriate treatments, and increased length of stay in hospitals (8). Oral reports also allow EMS crews to highlight and accentuate areas of concern during their independent role as providers to patients in the prehospital environment and during handoffs.

Our study showed several key results. The first major result being that data metrics such as chief complaint and history of present illness was most often transferred. Objective data such as vital signs and physical exams were less often transferred. The second major issue our study showed is that when considering the cases where EMS did not report vital signs initially, they were prompted for vital signs $26 \%$ of the time by ED staff. In each of the cases where they were asked for vital signs, they were able to provide an answer. Another key result was the comparison of medical vs trauma cases which showed a large discrepancy in the transmission of demographics, physical exam, and assessment which greatly favored EMS crews doing this during traumas. Interestingly, vital signs were verbally reported slightly less often during trauma handoffs. One possible explanation for this is that vital signs were communicated via radio report en route to the hospital for traumas or that most trauma patients are hooked up to monitoring equipment upon arrival to the $\mathrm{ED}$ and during most trauma handoffs. Finally, interruptions to transfers and repeated questions constituted a sizeable number of the handoffs occurring $20 \%$ and $9 \%$ of the time respectively.

Our study concurred with similar studies in that chief complaint was the most often transferred data point. ${ }^{5,6}$ The current literature illustrates vital signs were omitted $50-70 \%$ of the time and past medical history was omitted $40-60 \%$ of the time. ${ }^{5,6}$ Our study showed similar results in regard to omission of vital signs and past medical history. In addition, similar findings

Figure 3: Comparison of the percentage of time data metrics were transferred in trauma vs medical cases

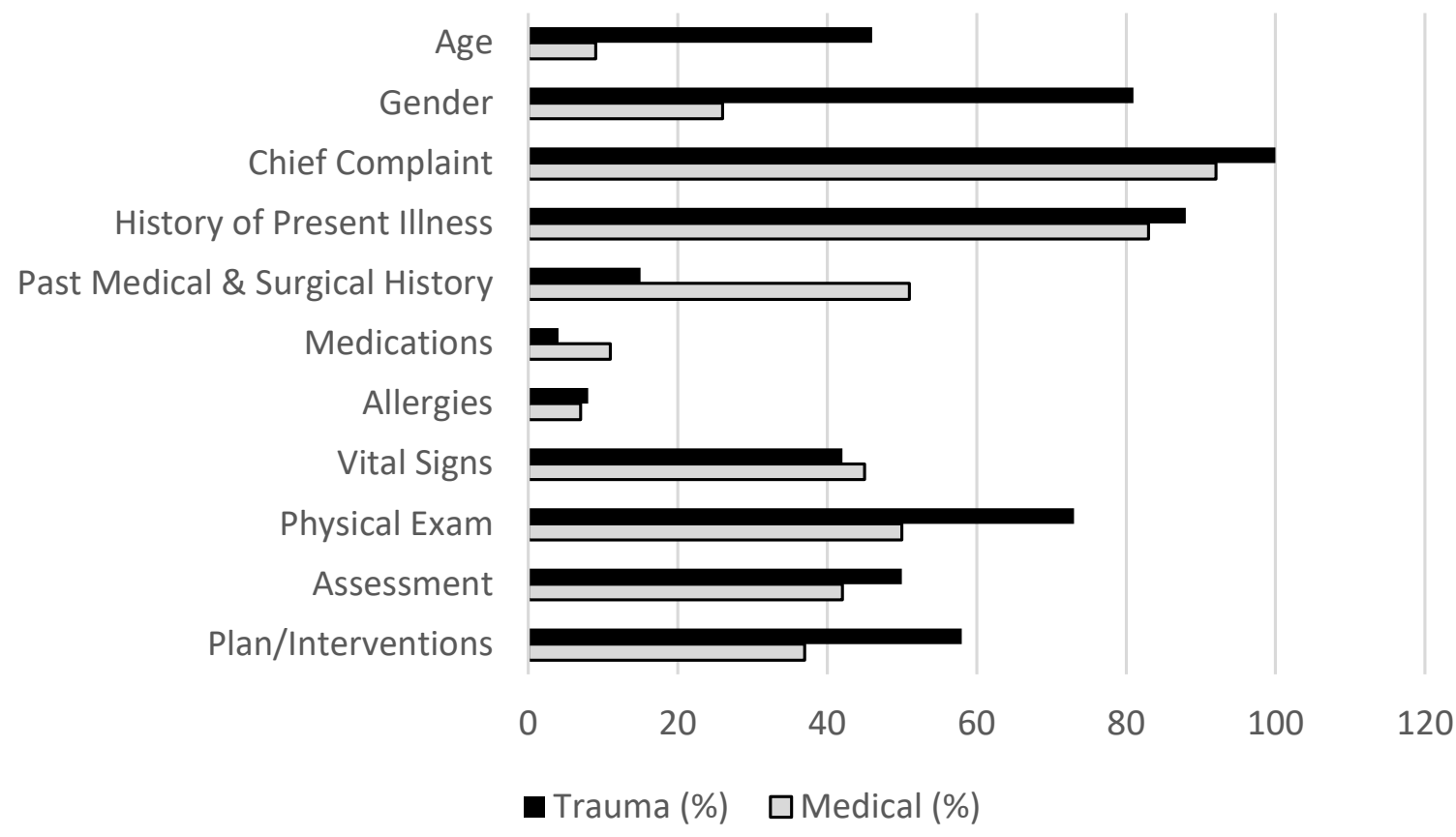

JCEMS · Volume $4 \cdot$ Issue $2 \cdot$ October 2021 
were observed concerning physical exam findings with a range of inclusion in handoffs $47-67 \%$ of the time. ${ }^{5,6}$

In contrast, the largest study $(\mathrm{n}=1091)$ available studying this same interaction showed that over $70 \%$ of the time EMS crews did report individual vital signs upon handoff. ${ }^{7}$ This represents a stark contrast with our study and the two mentioned previously. There are many possible explanations for this including but not limited to differences in state/local training of EMS and protocols within the specific ED. Another large contrast between our study and previous ones was the inclusion of age in handoffs. Whereas age was given in $19 \%$ of our handoffs it was given $41-84 \%$ of the time in other studies. ${ }^{5,6,7}$ When examining a subset of trauma patients, our findings are consistent with previous research, where age was reported $40 \%$ of the time. ${ }^{5}$ Interruptions and repeat questions occurred less often in our study when compared to another. ${ }^{6}$

Few studies have been conducted on the specific content of EMS handoffs. Those that have been performed previously have done so in individual locations such as ours. In general our data aligned with that of two other studies. As previous literature and review articles have pointed out there is a lack of data in this significant area of patient safety. ${ }^{9,10}$ Our data builds upon this already limited data while showing a general trend of incomplete patient handoffs. Objectively, we know that $80 \%$ of medical errors involve miscommunication during patient transfers and handoffs. ${ }^{8}$ Studies interviewing EMS personnel have shown that they have often felt the messages they are trying to relay to ED personnel are not heard, leading to perceived delays in care. ${ }^{11,12}$ Improvements in the relationship between EMS and ED personnel in the form of feedback have already shown significant improvement in the treatment of ischemic strokes. ${ }^{13}$ Documentation accompanying handoffs by way of a standardized checklist has been shown by a collegiate ambulance service to improve the completeness of handoffs. ${ }^{14}$ Understanding and improving this complex interaction between EMS and ED personnel is a modifiable patient safety concern for which better understanding is required.

Previous literature has highlighted the importance of structured tools and standardization of oral handoffs. ${ }^{9,10}$ Suggested improvements include better incorporation of electronic information boards with minimum requirements for patient handoffs in addition to interprofessional training so that providers from different backgrounds can better communicate and feel respected and listened to. ${ }^{10,12}$ Further studies could focus on implementing an interprofessional training program with local EMS providers and ED staff to ascertain if improvements can be made qualitatively. Studies could also question patient outcomes based on the quality of EMS handoffs.

\section{Limitations}

This study only took place between the hours of 0900-2000 Monday through Friday due to the observers' schedules. It is certainly possible that interactions could vary at different times of the day and late into the night, or during the weekends, leading specifically to selection bias.

During trauma bay resuscitations, it can be challenging to hear information, and some may have been missed by the observers.

Some information may have been transferred by a second EMS, by initial radio transmission, or by the patient, thereby being missed by the observer.

\section{Conclusion}

While patient reports from EMS to ED personnel frequently include chief complaint and history of the present illness other patient metrics are less commonly transferred. This represents possible weaknesses in the completeness of patient data received by ED personnel from EMS.

There appear to be opportunities to improve communication of certain key physical exam findings like vital signs.

Follow-up research could focus on ascertaining how the quality of EMS reports affects patient outcomes or on improving communication especially handoffs between EMS and the ED.

\section{Acknowledgements}

The authors report no conflicts of interest.

\section{References}

1. Rui P, Kang K, Ashman JJ. National Hospital Ambulatory Medical Care Survey: 2016 emergency department summary tables. 2016. Available from: https://www.cdc.gov/nchs/data/nhamcs/web_tables/2016_ed_web_tables. pdf

2. American College of Emergency Physicians, Emergency Nurses Association, National Association of EMS Physicians, National Association of Emergency Medical Technicians, National Association of State EMS officials. Transfer of patient care between EMS providers and receiving facilities. Prehosp Emer Care. 2014;18(2):503-503. doi:10.3109/10903127.2014.883001

3. United States. National Highway Traffic Safety Administration, and United States. Maternal Child Health Bureau. "First Responder: National Standard Curriculum (Instructor’s Course Guide)." (1995). Web.

4. Lindsay LP, White ML, Sawyer K, Pruitt C, Wang H. Pilot survey of components of emergency medical services emergent handoffs. Ann Emerg Med. 2013;62(4). doi:10.1016/j.annemergmed.2013.07.111

5. Goldberg SA, Porat A, Strother CG, et al. Quantitative analysis of the content of EMS handoff of critically ill and injured patients to the Emergency Department. Prehosp Emerg Care. 2016;21(1):14-17. doi:10.1080/10903127 .2016 .1194930

6. Sumner BD, Grimsley EA, Cochrane NH, et al. Videographic assessment of the quality of EMS to Ed Handoff communication during pediatric resuscitations. Prehosp Emerg Care. 2018;23(1):15-21. doi:10.1080/1090312 7.2018 .1481475

7. Panchal AR, Gaither JB, Svirsky I, Prosser B, Stolz U, Spaite DW. The impact of professionalism on transfer of care to the Emergency Department. J Emerg Med. 2015;49(1):18-25. doi:10.1016/j.jemermed.2014.12.062 
8. Joint Commission Center for Transforming Healthcare. Joint Commission Center for Transforming Healthcare Tackles Miscommunication Among Caregivers. Joint Commission Center for Transforming Healthcare, October $1,2017$.

9. Wood K, Crouch R, Rowland E, Pope C. Clinical handovers between Prehospital and Hospital Staff: Literature review. Emerg Med J. 2014;32(7):577581. doi:10.1136/emermed-2013-203165

10. Jensen SM, Lippert A, Østergaard D. Handover of patients: A topical review of ambulance crew to emergency department handover. Acta Anaesthesiol Scand. 2013;57(8):964-970. doi:10.1111/aas.12125

11. Meisel ZF, Shea JA, Peacock NJ, et al. Optimizing the patient handoff between Emergency Medical Services and the Emergency Department. Ann Emerg Med. 2015;65(3). doi:10.1016/j.annemergmed.2014.07.003

12. Bost N, Crilly J, Patterson E, Chaboyer W. Clinical handover of patients arriving by ambulance to a hospital emergency department: A qualitative study. Intern Emerg Nurs. 2012;20(3):133-141. doi:10.1016/j.ienj.2011.10.002

13. Choi B, Tsai D, McGillivray CG, Amedee C, Sarafin J-A, Silver B. Hospital-directed feedback to Emergency Medical Services improves prehospital performance. Stroke. 2014;45(7):2137-2140. doi:10.1161/ strokeaha.114.005679

14. Alatis A, Monahan B, Raymond A, Hudson K, Vieth J, Nable J. Checklists improve EMS Documentation: Quality Improvement in a collegiatebased EMS Agency. J Coll Emerg Med Serv. 2020;3(1):16-21. doi:10.30542/ jcems.2020.03.01.05

Author Affiliations: From University at Alabama at Birmingham - in Birmingham, AL (M.D.M, J.J.G., J.B., W.A.S.).

Address for Correspondence: Walter A. Schrading, MD, FACEP, FAWM | Email: wschrading@uabmc.edu
Conflicts of Interest/Funding Sources: By the JCEMS Submission Declaration Form, all authors are required to disclose all potential conflicts of interest and funding sources. All authors declared that they have no conflicts of interest. All authors declared that they did not receive funding to conduct the research and/or writing associated with this work.

Authorship Criteria: By the JCEMS Submission Declaration Form, all authors are required to attest to meeting the four ICMJE.org authorship criteria: (1) Substantial contributions to the conception or design of the work; or the acquisition, analysis, or interpretation of data for the work; AND (2) Drafting the work or revising it critically for important intellectual content; AND (3) Final approval of the version to be published; AND (4) Agreement to be accountable for all aspects of the work in ensuring that questions related to the accuracy or integrity of any part of the work are appropriately investigated and resolved.

Submission History: Received June 22, 2020; accepted for publication September $12,2021$.

Published Online: October 31, 2021

Published in Print: October 31, 2021 (Volume 4: Issue 2)

Reviewer Information: In accordance with JCEMS editorial policy, Original Research manuscripts undergo double-blind peer-review by at least two independent reviewers. JCEMS thanks the anonymous reviewers who contributed to the review of this work.

Copyright: @ 2021 Monaco, Grove, Beckedorf \& Schrading. This is an open access article distributed under the terms of the Creative Commons Attribution 4.0 International (CC BY 4.0) License, which permits unrestricted use, distribution, and reproduction in any medium, provided the original author and source are credited. The full license is available at: https://creativecommons.org/licenses/ by $/ 4.0 /$

Electronic Link: https://doi.org/10.30542/JCEMS.2021.04.02.03 


\title{
Determinants of Volunteer Collegiate-Based Emergency Medical Service Budget Size
}

\author{
Murray J. Bartho, BS, EMT; Tom Goode, MS, EMT; Gabe Gan, MPH, NRP
}

\section{ABSTRACT}

Background: Collegiate-based emergency medical services (CBEMS) agencies are a unique service model within the world of emergency medical services (EMS). CBEMS agencies vary widely concerning organizational, operational, and other characteristics. The financial setups of these groups are also varied, but not well documented. Objectives: The purpose of this study was to examine determinants of budget size and budget sources for a cross-section of CBEMS agencies. Methods: An electronic survey was sent to volunteer CBEMS agencies using the National Collegiate Emergency Medical Services Foundation (NCEMSF) database. The survey requested agency demographics and budget data. Results were collected and analyzed using a chi-square test. Results: Large student body populations, agencies that transport patients, 24/7 response agencies, and agencies with high annual call volumes were associated with larger budgets. Most CBEMS agencies received the bulk of their funds from their institution and few relied on fundraising. Conclusion: Many disparate factors affect CBEMS's budget size. CBEMS agencies exhibit high levels of diversity in budget size, operational characteristics, and fundraising strategies. Access to peer agency information will allow CBEMS agencies to build and maintain well-funded organizations.

Keywords: budget, administration, collegiate-based emergency medical services

Corresponding Author and Author Affiliations: Listed at the end of this article.

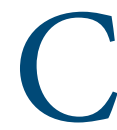
ollegiate-based emergency medical services (CBEMS) agencies play an important role in keeping campus communities healthy and safe by providing quality emergency medical treatment. Like the colleges and universities they serve, CBEMS agencies are unique. Their idiosyncrasies have been captured in past surveys as well as by the EMS Organization Database hosted on the National Collegiate Emergency Medical Services Foundation (NCEMSF) website. ${ }^{1-6}$ Some agencies transport their patients via ambulance, while others escalate care to on-site advanced life support (ALS) services or call 911. Some are staffed by emergency medical technicians (EMTs), while a minority employ paramedics. ${ }^{5}$ Annual call volume can range from less than 100 to over $1,000 .^{5}$

CBEMS agencies also differ with respect to budget size and source., ${ }^{5,7}$ Literature has shown that CBEMS agency budget size has a large range. ${ }^{5,7}$ Funding variations could have real-world consequences, specifically if agency operations are constrained by a lack of funds. Funding problems can damage emergency medical services (EMS) systems in general, and CBEMS systems in particular. $^{8}$

Murray J. Bartho, BS, EMT, is a hearing loss researcher at Stanford University and volunteer EMT with Stanford EMS. He has served as Director of Logistics for Stanford EMS and as Operations Officer for Santa Clara University EMS. Tom Goode, MS, EMT, works as a statistical analyst at the RAND Corporation and as an EMT with the Munhall Area Prehospital Services. Gabe Gan, MPH, NRP, works as a management and program analyst at the DC Fire and EMS Department. $\mathrm{He}$ is a volunteer Paramedic with the Silver Spring Volunteer Fire Department.
For example, budget cuts are correlated with an increased mean response time for CBEMS agencies ${ }^{7}$ Budget sources for CBEMS agencies have been investigated, but the percentage contribution to the total budget of individual sources has not. ${ }^{4}$

This effort builds on previous demographic surveys to describe the financial details of surveyed CBEMS agencies in more depth. The goals of this project are to begin a detailed discussion on CBEMS funding and to serve as a reference for new or existing CBEMS agencies looking to contextualize their finances.

\section{Methods}

The survey was developed using Google Forms and distributed electronically to CBEMS agencies listed on the NCEMSF United States EMS Organizations webpage through 11 NCEMSF Regional Coordinators. ${ }^{6}$ Through this method, 1 additional CBEMS agency not listed on the NCEMSF webpage responded to the survey. In parallel, 165 target agencies were contacted at least twice over 4 months (Figure 1). After contact, 105 organizations were rendered ineligible, most (96 agencies, 58\%) because they failed to complete the survey. 60 organizations completed the survey, for a response rate of $36 \%$, with 5 organizations excluded from analysis. 55 agencies made up the study (Figure 2).

Historically, it has been difficult to survey the CBEMS community due to personnel turnover. Emphasis was placed on making 
Figure 1: CBEMS agencies were identified and screened before survey release, and again post-survey. Reasons for exclusion are enumerated at each screening step. This process was based on the PRISMA method. ${ }^{16}$

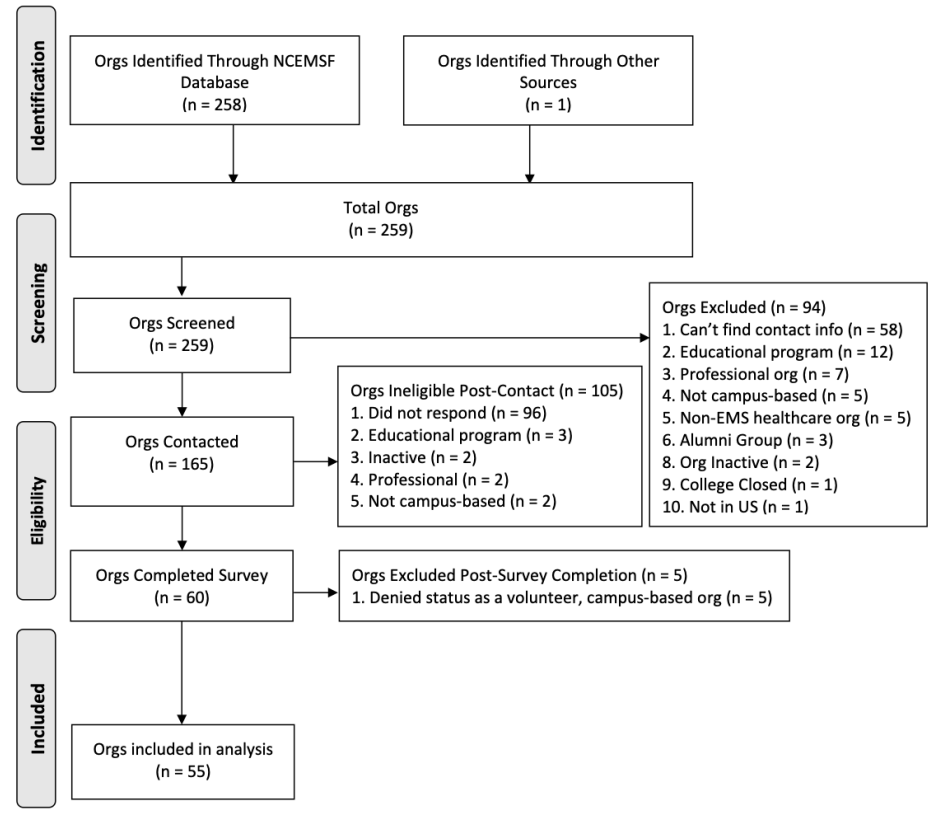

the survey brief to increase response rates. ${ }^{9}$ Professional EMS agencies were screened out by survey respondents affirming they belonged to an all-volunteer agency. The full survey transcript can be found in Supplementary Materials. Survey respondent privacy is protected by only publishing anonymized statistics. IRB review of this project was deemed not necessary by the Research Compliance Office at Stanford University.

Graphical representations were created using Microsoft Excel.
Chi-square analyses were performed using R. Frequency rates are often shown as percentages of the total amount in that category, to account for different amounts of respondents in each category. Statistical significance was set at $\mathrm{p}<0.05$.

\section{Results}

\section{CBEMS Agency Characteristics}

Demographics of survey respondents are seen in Table 1 . The vast majority operated at basic life support (BLS) or intermediate life support (ILS) levels, with $4 \%$ providing ALS. Two-thirds were non-transporting services, while one-third provided medical transport. CBEMS agencies were divided into three bins based on their campus student population. ${ }^{10}$ Annual call volume was measured. Agencies were allowed to select multiple types of coverage hours.

\section{Campus Student Body and Budget Size}

Student body size was significantly related to budget size, $\mathrm{X} 2(6$, $\mathrm{N}=55)=23.7, \mathrm{p}<.001$ (Figure 3). Generally, large campus CBEMS agencies had the largest budgets, followed by medium campuses, then small. Large campus groups had a bimodal budget distribution, with the majority of groups (60\%) over $\$ 45,000$ annually, and 33\% accessing less than $\$ 15,000$. Medium CBEMS agencies had a more balanced distribution. The most common budget bin for agencies at medium-sized schools was $\$ 0$ - $\$ 14,999$, with $38 \%$ of agencies at this size. Besides that bin, none other captured more than $30 \%$ of medium-size school CBEMS agencies. CBEMS agencies at small schools generally had the smallest budgets, none accessing more than $\$ 30,000$ annually, and $74 \%$ having budgets smaller than $\$ 15,000$.

Figure 2: Regional locations of the 55 CBEMS agencies in the study. Boxed numbers are the number of agencies in that region recruited to the study. Legend indicates the total agencies per region in the NCEMSF US database. Figure created using mapchart.net

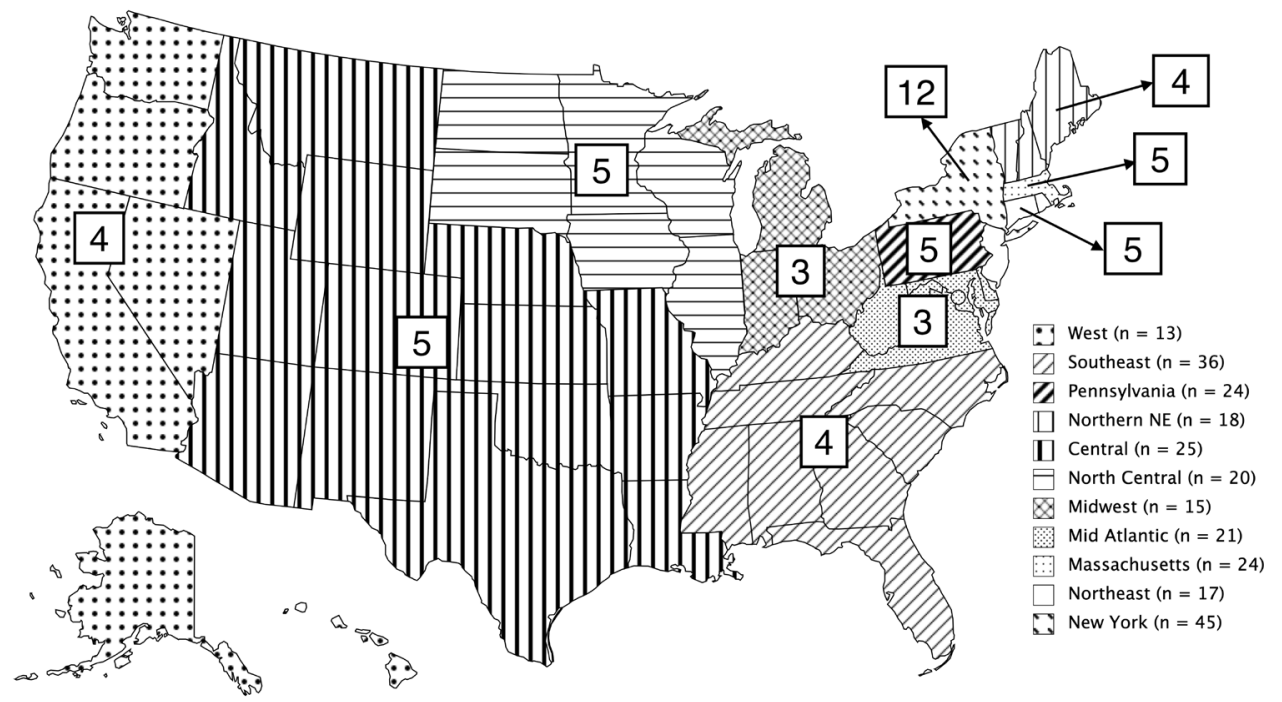


Table 1: Survey respondent demographics are shown as values and percentages. CBEMS agencies that skipped answering a question or the question didn't apply to their organization were not counted. Respondents with multiple answers to a question were counted for each answer.

\begin{tabular}{|c|c|c|c|}
\hline \multicolumn{2}{|c|}{ Organizational Descriptor } & \multicolumn{2}{|c|}{$\mathrm{N}=\mathbf{5 5}$} \\
\hline \multirow{3}{*}{ Campus Environs } & City/Urban & 32 & (58) \\
\hline & Suburban & 12 & (22) \\
\hline & Rural & 11 & (20) \\
\hline \multirow{2}{*}{ Level of Care } & BLS/ILS & 53 & (96) \\
\hline & ALS & 2 & (4) \\
\hline \multirow{2}{*}{ Transporting Status } & Transporting & 18 & (33) \\
\hline & Non-transporting & 37 & (67) \\
\hline \multirow{3}{*}{ Student Body Size } & Small & 19 & (35) \\
\hline & Medium & 21 & (38) \\
\hline & Large & 15 & (27) \\
\hline \multirow{5}{*}{$\begin{array}{l}\text { Annual Call } \\
\text { Volume }\end{array}$} & $<100$ calls & 14 & (25) \\
\hline & $100-300$ calls & 19 & (35) \\
\hline & $300-500$ calls & 13 & (24) \\
\hline & $500-1,000$ calls & 5 & (9) \\
\hline & $>1,000$ calls & 4 & (7) \\
\hline \multirow{3}{*}{ Coverage Hours } & $24 / 7$ & 34 & $(62)$ \\
\hline & Partial & 14 & $(25)$ \\
\hline & Event-Based & 7 & (13) \\
\hline \multirow{4}{*}{ Annual Budget } & $\$ 0-\$ 14,999$ & 27 & (49) \\
\hline & $\$ 15,000$ - $\$ 29,999$ & 12 & (22) \\
\hline & $\$ 30,000-\$ 44,999$ & 3 & (5) \\
\hline & $>\$ 45,000$ & 13 & (24) \\
\hline \multirow{5}{*}{$\begin{array}{l}\text { Budget Sourced } \\
\text { from Institution }\end{array}$} & $0 \%-20 \%$ & 7 & (13) \\
\hline & $21 \%-40 \%$ & 3 & (5) \\
\hline & $41 \%-60 \%$ & 0 & (0) \\
\hline & $61 \%-80 \%$ & 3 & (5) \\
\hline & $81 \%-100 \%$ & 42 & (76) \\
\hline \multirow{5}{*}{$\begin{array}{l}\text { Budget Sourced } \\
\text { from Fundraising }\end{array}$} & $0 \%-20 \%$ & 46 & (84) \\
\hline & $21 \%-40 \%$ & 3 & (5) \\
\hline & $41 \%-60 \%$ & 2 & (4) \\
\hline & $61 \%-80 \%$ & 1 & (2) \\
\hline & $81 \%-100 \%$ & 3 & (5) \\
\hline
\end{tabular}

Figure 3: Student Body and Budget Size. Size of the tertiary educational institution related to budget size of the CBEMS agency that serves its campus. Colleges and universities were split into 3 size categories based on the total student population. ${ }^{10}$ Groups were plotted as frequency percentages within that size category (see Methods).

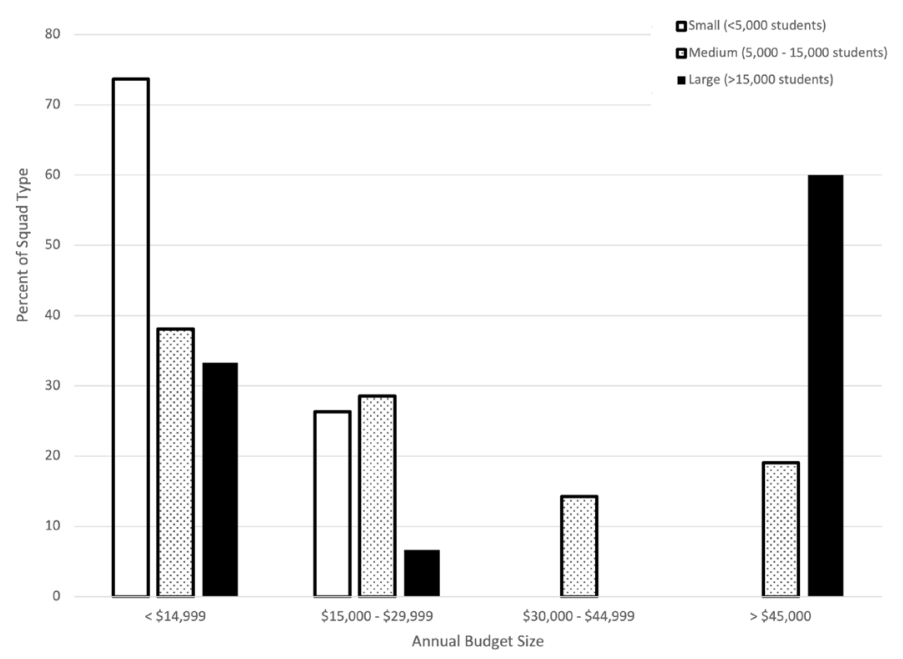

Figure 4: Transportation Status and Budget Size. Budget size of transporting and non-transporting agencies. Transporting agencies were defined as those who can take their patients directly to the hospital. Data are plotted as frequency percentages within each category (see Methods).

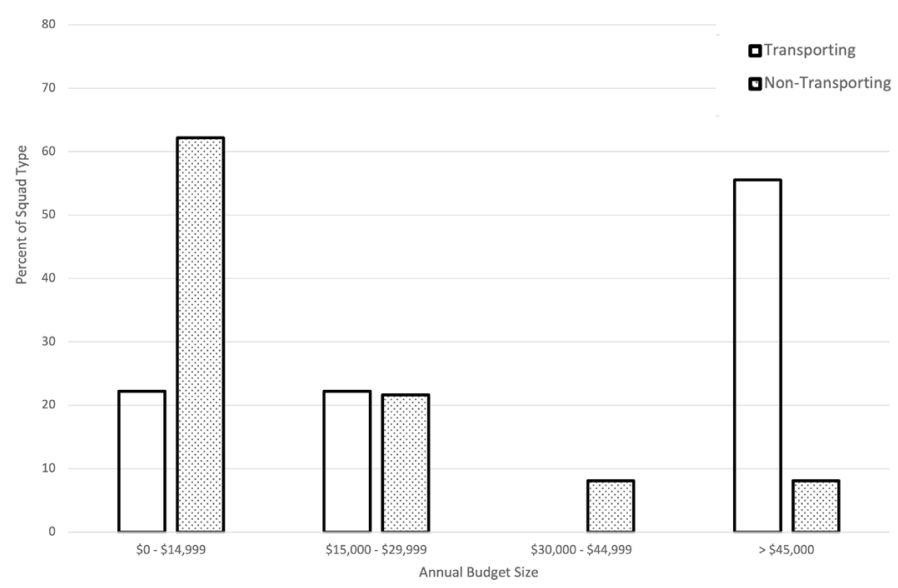

Transporting versus Non-Transporting Agencies

Transportation status was significantly related to budget size, X2(3, $\mathrm{N}=55)=16.9, \mathrm{p}<.001$ (Figure 4 ). Transporting agencies generally had larger budgets than non-transporting agencies. Most (56\%) transporting agencies had budgets greater than $\$ 45,000$. Only $8 \%$ of non-transporting agencies had budgets of this size. Most (62\%) non-transporting agencies had budgets less than $\$ 15,000$. Middling budget sizes, between $\$ 15,000$ - $\$ 45,000$, saw closer rates of transporting (22\%) and non-transporting (30\%) agencies. 
Figure 5: Call Volume and Budget Size. Call volume of the CBEMS agency related to its budget size. CBEMS agencies were split into 5 populations based on call volumes. Data are plotted as frequency percentages within each call volume category (see Methods).

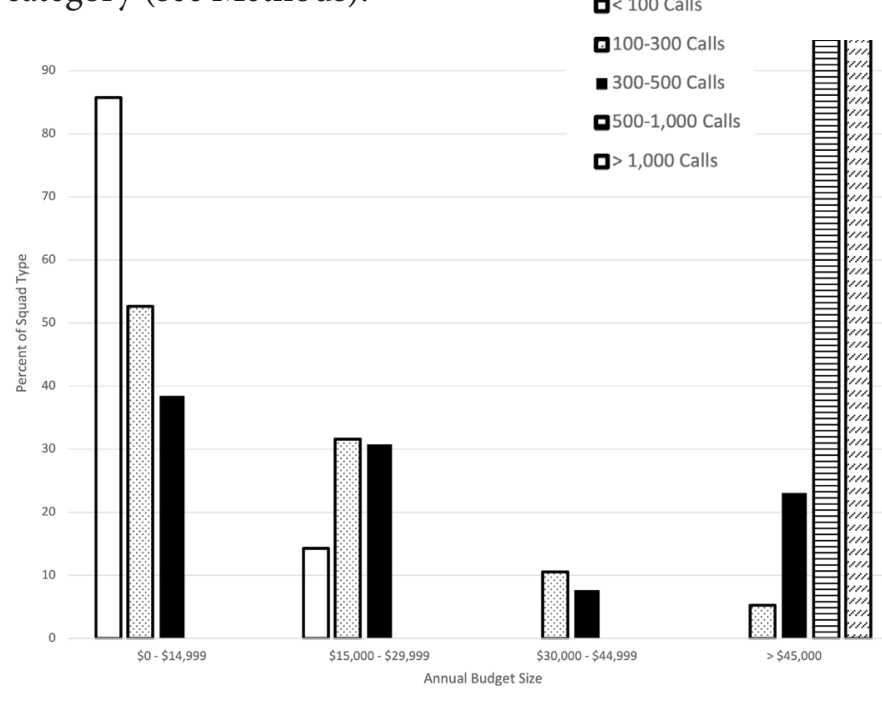

Call Volume and Budget Size

Call volume was significantly related to budget size, $\mathrm{X} 2(12, \mathrm{~N}=$ $55)=42.7, \mathrm{p}<.001$ (Figure 5). Budget size generally increased with call volume for CBEMS agencies. All agencies in the two highest call volume brackets also had budgets over $\$ 45,000$. Agencies with 300-500 calls were more evenly distributed, with no one budget category having more than $40 \%$ of those agencies. 100-300 call agencies all had budgets under $\$ 40,000$, except for one outlier with a budget over $\$ 100,000$. Agencies with less than 100 calls all had budgets under $\$ 20,000$.

\section{Coverage Hours and Budget Size}

Coverage hour schedules are significantly related to budget size, $\mathrm{X} 2(6, \mathrm{~N}=55)=14.0, \mathrm{p}=.03$ (Figure 6). Generally, the more coverage hours a CBEMS agency offers, the larger its budget. Only $32 \%$ of $24 / 7$ agencies have budgets under $\$ 15,000$, compared to $67 \%$ of partial hours squads and $86 \%$ of event-based groups. In the middling budget range, between $\$ 15,000$ and $\$ 45,000$, there was an almost equal distribution between 24/7 (32\%) and partial hours (33\%) groups. At the high end, with budgets greater than $\$ 45,000$, the largest group of $24 / 7$ squads existed (35\%).

\section{Source of Budget Funds}

CBEMS agencies received most, if not all, of their funding from their higher education institution, and fundraised very little for their annual budget (Figure 7). Most (76\%) CBEMS agencies got $81 \%-100 \%$ of their budget from their institution, and $84 \%$ had to fundraise only $0 \%-20 \%$ of their annual budget.
Figure 6: Coverage Hours and Budget Size. Budget sizes of CBEMS agencies with different coverage hours are shown. The coverage hours represent aggregated survey bin options: 24/7 (24/7 calendar year, 24/7 academic year); partial hours (nightly, nightly calendar year, nightly academic year, limited daily hours academic year, limited daily hours calendar year, weekends academic year, weekends calendar year); events (event-based). Groups were plotted as frequency percentages within each coverage category (see Methods).

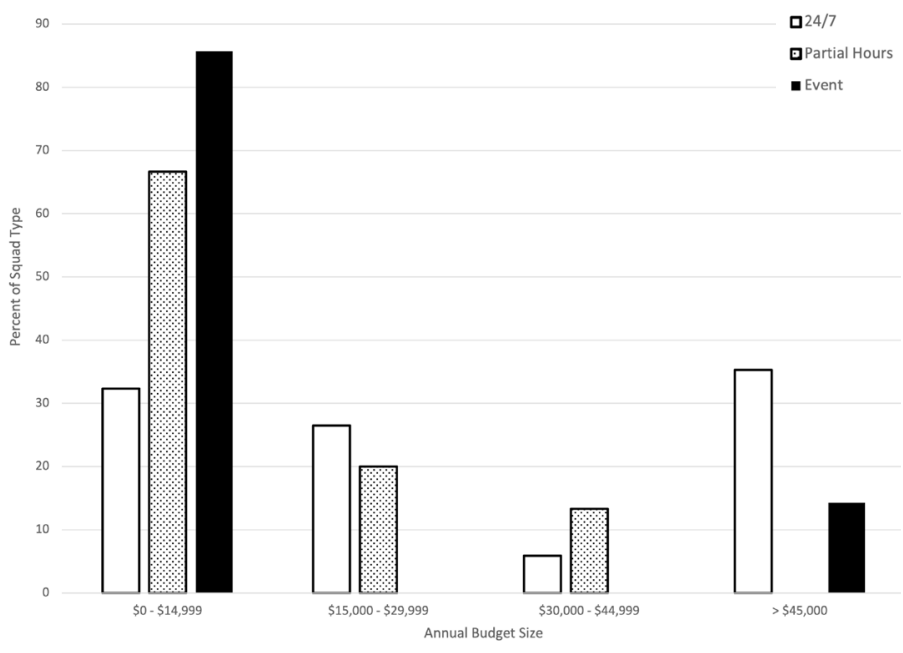

Figure 7: Budget Source. The percentage of CBEMS budgets coming from the institution itself and grassroots fundraising. Institutional funding includes all funds supplied by the college or university itself, while grassroots fundraising includes funds gathered from teaching CPR classes, soliciting donations, or other acts undertaken by the CBEMS agency to collect money.

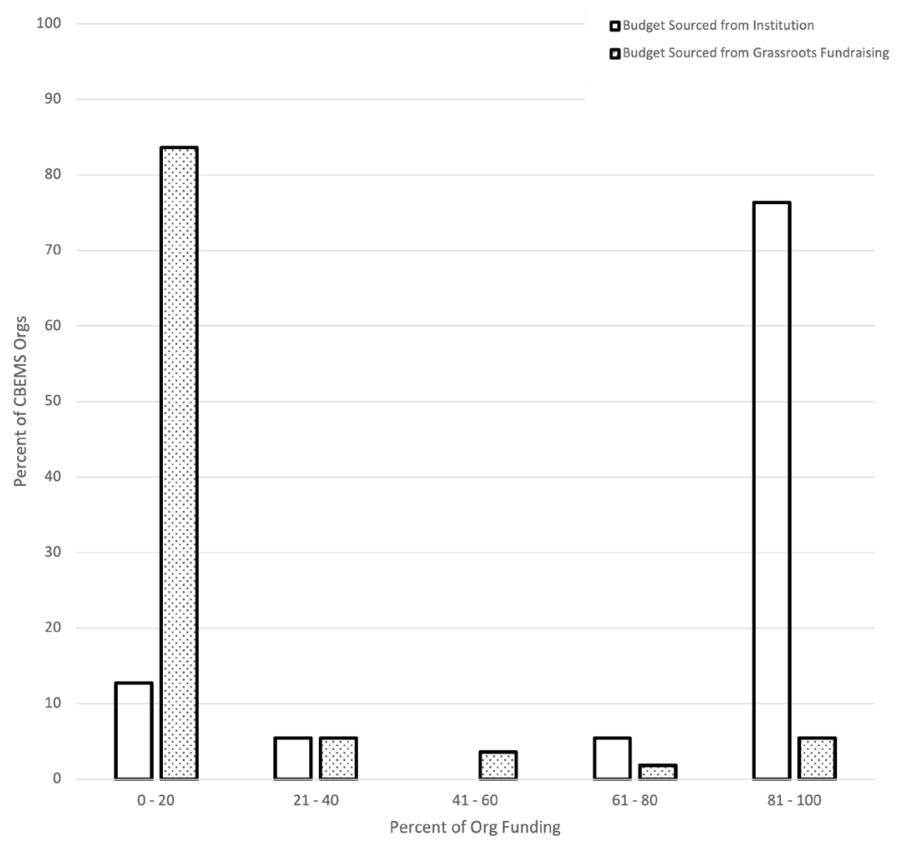

JCEMS · Volume $4 \cdot$ Issue $2 \cdot$ October 2021 


\section{Discussion}

Like the field of EMS as a whole, the origins of collegiate EMS are relatively recent. CBEMS agencies first formed in the emergency medical boom of the late 20th century, with limited information available on early groups. ${ }^{111-13}$ Initially without a national guiding organization, almost no literature existed discussing the details of CBEMS operation. ${ }^{14}$ Present-day diversity in CBEMS financials could be linked to limited research during the field's inception. Illustrative of this is the CBEMS mean annual budget of $\$ 39,333.38$ $(\mathrm{STD}=\$ 106,217.20, \mathrm{n}=101){ }^{5}$ The large standard deviation suggests variety in budget size. Our data show a relationship between CBEMS budget size and student population, with the most populated campuses having the highest funded CBEMS agencies. Serving a larger student body could involve higher call volume, larger squad size and campus area, and corresponding equipment and vehicle costs.

Similar explanations could be behind our other findings. Our results suggest that transporting agencies generally have higher budgets than non-transporting agencies. Owning, maintaining, staffing, and fueling a transport vehicle can come at a high cost, a potential cause for this relationship. ${ }^{15}$ Our data show that budget size generally increased with call volume. Responding to calls requires manpower, equipment, and being in service, all of which can impact the bottom line. 24-hour service tends to be the most expensive coverage scheme, with 24/7 squads in service for longer than their partial hours or event-based colleagues. Longer hours require more staffing and could be tied to an increase in patients seen due to a larger temporal catchment.

Funding from the university generally provided most or all monies for many CBEMS budgets, consistent with prior findings. ${ }^{4}$ Within higher education, there are many ways to secure funding. Some organizations are paid via the general fund (tuition), health fees, student fees, or operating budgets of the campus health office or department of public safety. ${ }^{14}$

The aforementioned trends appear to link together consistently. Larger budgets are linked with agencies who provide more medical care, whether that is through more service hours, a higher call volume, or offering transport, among other factors. While these data cannot discern causality, and said factors seem to be closely interconnected, it seems that the size of an agency's budget is to a certain degree a function of the scope of the agency's role in the community. One presumed causal relationship could be agencies who expand the scope of their operations are rewarded with attention and funding from their institution. Conversely, additional funding could pre-empt and support the expansion of CBEMS operations. Therefore, CBEMS agencies looking to establish or grow their service have options in discussions with administration. They could offer additional services to justify increased budgets in the future. They could also show the administration what additional funds would allow them to accomplish. We encourage CBEMS agencies and college administrators to use these data when discussing the desired role and scope of an agency, and the commensurate budget needs.

These results suggest the need for further research and comparison across the financial landscape of collegiate EMS. In higher education and healthcare, comparison between peer institutions is used to create standards, ensure competition and innovation, and drive the field forward. Research that looks into fundraising models (donations, CPR classes, etc.), gets granular non-binned data, or considers other variables (organization membership size, fee for service, etc.) is welcomed. Low survey completion rates due to incorrect contact information suggest that an update of the NCEMSF US Organization Database would prove useful. Allowing organizations to easily contact each other, build bonds, share information, and conduct research, would grow the NCEMSF on a national scale, and improve local CBEMS agencies. Healthy finances are important for CBEMS agencies, as underfunding can impact the ability of CBEMS agencies to provide quality emergency medical care. ${ }^{7}$ Hopefully, these results will serve as a useful guide for existing or emergent CBEMS agencies, leadership teams, school administrators, and other stakeholders to refer to when discussing finances.

\section{Limitations}

This work adds to a limited base of CBEMS budget research. While not definitive, our study encourages further investigation in the field. Results cannot be extrapolated to professional agencies or groups outside the US. The scope of this study did not include analysis of the full financial complexity of a CBEMS budget. Volunteer agencies often receive valuable non-monetary contributions from their institution or outside sources that were not measured in this survey. ${ }^{1,11}$ These contributions could include free or discounted operational real estate on campus grounds, training, uniforms, and food.

Sample size and respondent bias also limit this work (Figure 1). It is likely the 55 respondents are not perfectly representative of all-volunteer CBEMS agencies. Well-funded and established CBEMS agencies with dedicated websites, social media accounts, and permanent email addresses, may have been more likely to be reached by the survey. Agencies in flux or out of service due to the COVID-19 pandemic were less likely to answer the survey. Difficulty in contacting NCEMSF agencies calls for comprehensive efforts to gather updated demographic and contact information.

Binning of survey data limits the statistical interpretations that can be performed. Data could be skewed within a bin. Binning was a calculated decision in survey design that encouraged survey completion by making the survey faster to complete. ${ }^{9}$ While the trends are well illustrated by the histograms seen in this paper, further studies using continuous values could elucidate more specific information about CBEMS budgets.

\section{Conclusion}

CBEMS agencies have budgets that are as unique and diverse as 
the organizations themselves. A demographically widespread group of CBEMS agencies were surveyed about their budget size and sources of funding. Larger student populations, longer coverage hours, transporting agencies, and higher call volumes were more often served by CBEMS agencies with larger budgets. Inversely, smaller student populations, limited coverage times, non-transporting agencies, and low call volumes were associated with lower budgets. CBEMS agencies commonly receive most or all of their budget funds from their host institution, and rarely fundraise a large part of their budget. Small sample sizes, survey respondent bias, and non-financial budgetary elements limit the interpretability of this study. The importance of adequate funding for CBEMS agencies necessitates that agencies have better access to peer agency information to build and maintain quality organizations that continue to deliver high-value care to campus communities.

\section{Acknowledgements}

The authors would like to thank all survey participants for their diligence in providing accurate information, Michael Beautyman for his assistance in compiling the research team, and Patrick Atkinson for advice on statistical methods. The authors declare no financial support for the research, authorship, or publication of this article. The authors declare no conflicts of interest.

\section{References}

1. Wang J. Investigating the Current Status of Collegiate Emergency Medical Services (EMS) Organizations via a National Survey Study. ASU Electronic Theses and Dissertations. 2019.

2. King BR, Zachariah BS, Cone DC, Clark P. A survey of emergency medical services systems on college and university campuses. Prehospital Disaster Medicine. 1996;11(4):265-269. doi:10.1017/s1049023x00043107

3. Fisher J, Ray A, Milliron ME, Koenig GJ. Campus Based EMS: A Survey by the National Collegiate EMS Foundation. Oral Presentations of the Mediterranean Emergency Medicine Congress. 2003:6.

4. Fisher J, Ray A, Savett SC, Milliron ME, Koenig GJ. Collegiate-based emergency medical services (EMS): A survey of EMS systems on college campuses. Prehospital Disaster Medicine. 2006;21(2):91-96. doi:10.1017/ s1049023x00003411

5. Wolbrom DH, Rahman AM, Hilton MT. A Survey of 200 National Collegiate Emergency Medical Service Organizations. Prehospital Disaster Medicine. 2017;32(S1):S170-S171. doi:10.1017/S1049023X17004575

6. EMS Organization Database. National Collegiate Emergency Medical Services. Accessed January 15, 2021

7. D.H. W, Rahman AM, M.T. H. Underfunding of collegiate emergency medical services strains the delivery of adequate prehospital care. Academic Emergency Medicine. 2017;24:S230-S231. doi:10.1111/acem.13203

8. Heightman AJ. Understanding Why EMS Systems Fail. Journal of Emergency Medical Services. 2015;40(2).

9. Kost RG, Correa da Rosa J. Impact of survey length and compensation on validity, reliability, and sample characteristics for Ultrashort-, Short-, and Long-Research Participant Perception Surveys. Journal of Clinical and Translational Science. 2018;2(1):31-37. doi:10.1017/cts.2018.18.
10. College Sizes: Small, Medium, or Large. College Data. Accessed January 21, 2021.

11. Struve OAM. Design and Implementation of a Sustainable, University-Based, Emergency Medical Response Service. USF Theses Digital Repository. 2014.

12. Accidental Death and Disability: The Neglected Disease of Modern Society. National Academy of Sciences and National Research Council. 1966. doi:10.17226/9978.

13. Mission and History. National Collegiate Emergency Medical Services Foundation. Accessed January 15, 2021.

14. Delorenzo RA, Gray BC. Emergency medicine on college campuses: Key elements in the establishment and -success of a campus-based ambulance corps. Journal of American College Health Association. 1986;35(2):92-93. doi: $10.1080 / 07448481.1986 .9938968$

15. Lindberg, A., 2011. How Much Does an Ambulance Cost?. Tampa Bay Times. Accessed 26 February 2021.

16. Moher D, Liberati A, Tetzlaff J, Altman DG. Preferred Reporting Items for Systematic Reviews and Meta-Analyses: The PRISMA Statement. PLoS Medicine. 2009;6(7). doi:10.1371/journal.pmed.1000097

Author Affiliations: From Stanford University School of Medicine - in Stanford, CA (M.J.B.). From RAND Corporation; Munhall Area Prehospital Services - in Homestead, PA (T.G.). From DC Fire and EMS Department; Silver Spring Volunteer Fire Department - in Washington, D.C.; Silver Spring, MD (G.G.).

Address for Correspondence: Murray J. Bartho, BS, EMT | Email: mbartho@ stanford.edu | Phone: +1 (503) 729-2011 | Mailing Address: 3620 Olson Court, Lake Oswego, OR 97034

Conflicts of Interest/Funding Sources: By the JCEMS Submission Declaration Form, all authors are required to disclose all potential conflicts of interest and funding sources. All authors declared that they have no conflicts of interest. All authors declared that they did not receive funding to conduct the research and/or writing associated with this work.

Authorship Criteria: By the JCEMS Submission Declaration Form, all authors are required to attest to meeting the four ICMJE.org authorship criteria: (1) Substantial contributions to the conception or design of the work; or the acquisition, analysis, or interpretation of data for the work; AND (2) Drafting the work or revising it critically for important intellectual content; AND (3) Final approval of the version to be published; AND (4) Agreement to be accountable for all aspects of the work in ensuring that questions related to the accuracy or integrity of any part of the work are appropriately investigated and resolved.

Submission History: Received March 2, 2021; accepted for publication September 3, 2021.

Published Online: October 31, 2021

Published in Print: October 31, 2021 (Volume 4: Issue 2)

Reviewer Information: In accordance with JCEMS editorial policy, Original Research manuscripts undergo double-blind peer-review by at least two independent reviewers. JCEMS thanks the anonymous reviewers who contributed to the review of this work.

Copyright: (๑) 2021 Bartho, Goode \& Gan. This is an open access article distributed under the terms of the Creative Commons Attribution 4.0 International (CC BY 4.0) License, which permits unrestricted use, distribution, and reproduction in any medium, provided the original author and source are credited. The full license is available at: https://creativecommons.org/licenses/by/4.0/

Electronic Link: https://doi.org/10.30542/JCEMS.2021.04.02.04 



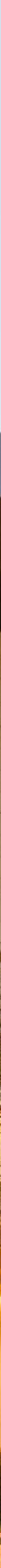

